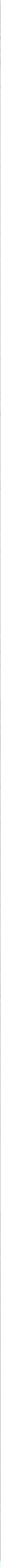




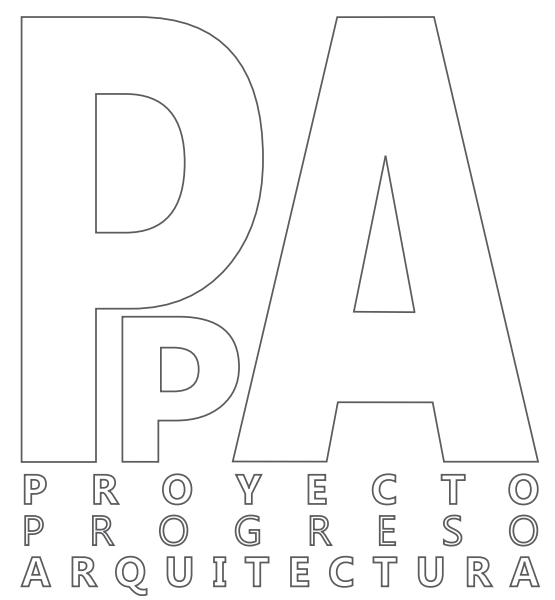

\section{EMERGENCIAS DEL ESPACIO COMÚN}

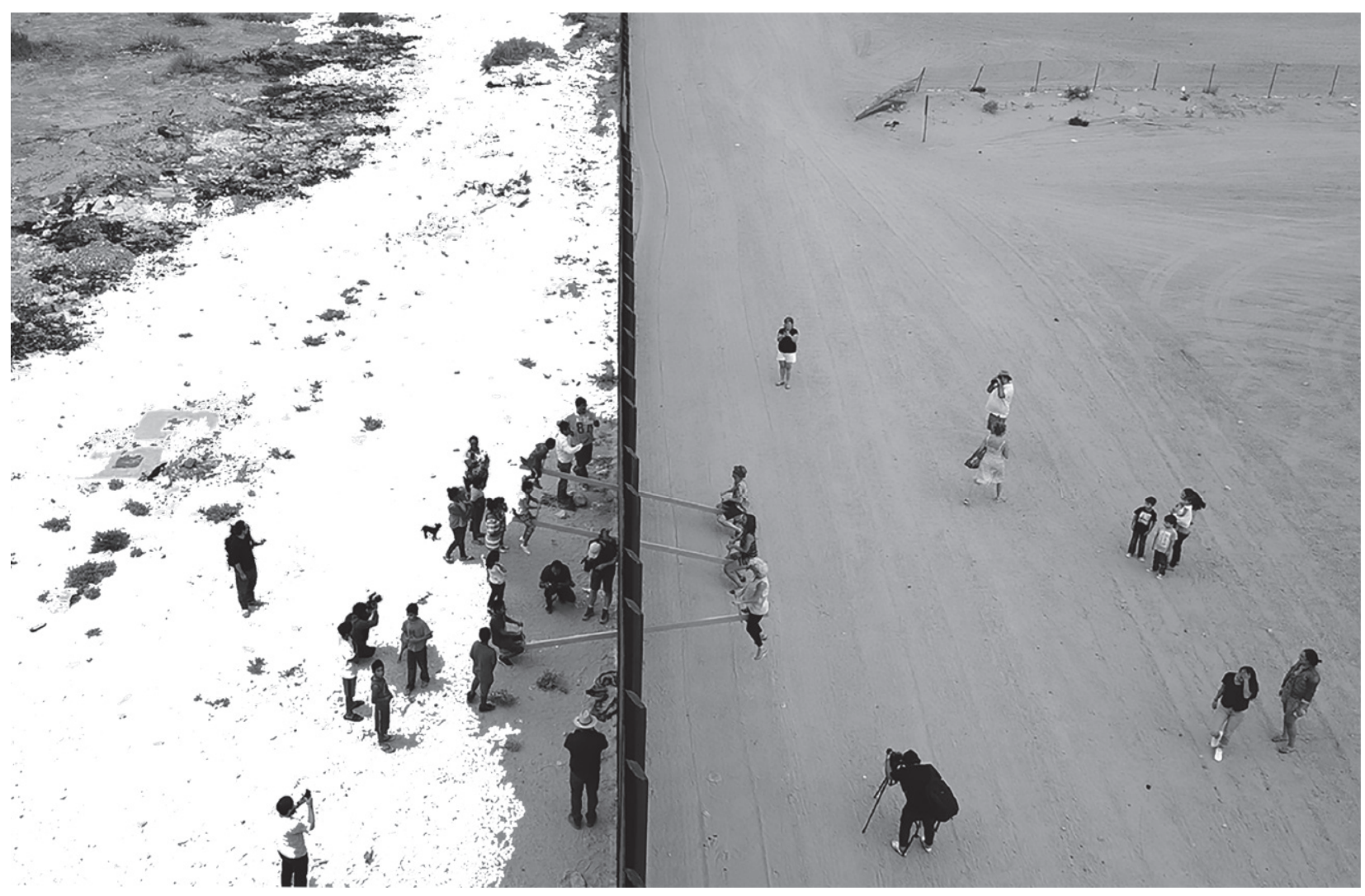

EDITORIAL UNIVERSIDAD DE SEVILLA AÑO 2019. ISSN 2171-6897 ISSNe 2173-1616 DOI: http://dx.doi.org/10.12795/ppa 


\section{REVISTA PROYECTO PROGRESO ARQUITECTURA}

\section{N255}

\section{emergencias del espacio común}
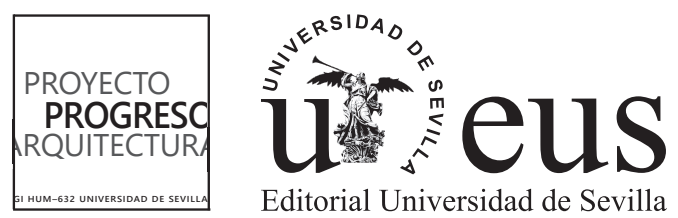


\section{emergencias del espacio común}

EDITA

Editorial Universidad de Sevilla. Sevilla

DIRECCIÓN CORRESPONDENCIA CIENTÍFICA

E.T.S. de Arquitectura. Avda Reina Mercedes, nº $241012-$

Sevilla.

Amadeo Ramos Carranza, Dpto. Proyectos Arquitectónicos.

e-mail: revistappa.direccion@gmail.com

\section{EDICIÓN ON-LINE}

Portal informático https://revistascientificas.us.es/index.php/ppa Portal informático Grupo de Investigación HUM-632

http://www. proyectoprogresoarquitectura.com

Portal informático Editorial Universidad de Sevilla http://www. editorial.us.es/

(c) EDITORIAL UNIVERSIDAD DE SEVILLA, 2019.

Calle Porvenir, 27. 41013 SEVILLA. Tfs. 954487447 / 954487451

Fax 954487443. [eus4@us.es] [http://www.editorial.us.es]

(c) TEXTOS: SUS AUTORES,

(C) IMÁGENES: SUS AUTORES Y/O INSTITUCIONES

DISEÑO PORTADA:

Rosa María Añón Abajas - Amadeo Ramos Carranza

En base a la fotografía: Cortesía y (c) Rael San Fratello, 2019.

DISEÑO PLANTILLA PORTADA-CONTRAPORTADA

Miguel Ángel de la Cova Morillo-Velarde

DISEÑO PLANTILLA MAQUETACIÓN

Maripi Rodríguez

MAQUETACIÓN

Referencias Cruzadas

CORRECCION ORTOTIPOGRÁFICA

Deculturas

ISSN (ed. impresa): 2171-6897

ISSN-e (ed. electrónica): 2173-1616

DOI: http://dx.doi.org/10.12795/ppa

DEPÓSITO LEGAL: SE-2773-2010

PERIOCIDAD DE LA REVISTA: MAYO Y NOVIEMBRE

IMPRIME: PODIPRINT

Reservados todos los derechos. Ni la totalidad ni parte de esta revista puede reproducirse o transmitirse por ningún procedimiento electrónico o mecánico, incluyendo fotocopia, grabación magnética o cualquier almacenamiento de información y sistema de recuperación, sin permiso escrito de la Editorial Universidad de Sevilla.

Las opiniones y los criterios vertidos por los autores en los artículos firmados son responsabilidad exclusiva de los mismos. 


\section{DIRECCIÓN}

Dr. Amadeo Ramos Carranza. Escuela Técnica Superior de Arquitectura. Universidad de Sevilla. España

\section{SECRETARÍA}

Dra. Rosa María Añón Abajas. Escuela Técnica Superior de Arquitectura. Universidad de Sevilla. España

\section{EQUIPO EDITORIAL \\ Edición:}

Dr. Amadeo Ramos Carranza. Escuela Técnica Superior de Arquitectura. Universidad de Sevilla. España.

Dra. Rosa María Añón Abajas. Escuela Técnica Superior de Arquitectura. Universidad de Sevilla. España.

Dr. Francisco Javier Montero Fernández. Escuela Técnica Superior de Arquitectura. Universidad de Sevilla. España.

Dra. Esther Mayoral Campa. Escuela Técnica Superior de Arquitectura. Universidad de Sevilla. España.

Dr. Miguel Ángel de la Cova Morillo-Velarde. Escuela Técnica Superior de Arquitectura. Universidad de Sevilla. España.

Dr. Germán López Mena. Escuela Técnica Superior de Arquitectura. Universidad de Sevilla. España.

Dra. Gloria Rivero Lamela. Escuela Técnica Superior de Arquitectura. Universidad de Sevilla. España.

Juan José López de la Cruz. Escuela Técnica Superior de Arquitectura. Universidad de Sevilla. España.

Guillermo Pavón Torrejón. Escuela Técnica Superior de Arquitectura. Universidad de Sevilla. España.

Externos edición (asesores):

Dr. José Altés Bustelo. Escuela Técnica Superior de Arquitectura. Universidad de Valladolid. España.

Dr. Carlos Arturo Bell Lemus. Facultad de Arquitectura. Universidad del Atlántico. Colombia.

Dr. José de Coca Leicher. Escuela Técnica Superior de Arquitectura. Universidad Politécnica de Madrid. España. Dra. Patricia de Diego Ruiz. Escuela Técnica Supeiror de Arquitectura y Geodesia. Universidad Alcalá de Heranes. España.

Dr. Alfonso del Pozo y Barajas. Escuela Técnica Superior de Arquitectura. Universidad de Sevilla. España.

Dr. Jaume J. Ferrer Fores. Escola Tècnica Superior

d'Arquitectura de Barcelona. Universitat Politècnica de Catalunya. España.

Dra. Laura MArtínez Guereñu. El School of Architecture \& Design, IE University, Madrid; Segovia. España.

Dra. Clara Mejía Vallejo. Escuela Técnica Superior de Arquitectura. Universidad Politécnica de Valencia. España.

Dra. Luz Paz Agras. Escuela Técnica Superior de Arquitectura. Universidade da Coruña. España.

Dra. Marta Sequeira. CIAUD, Faculdade de Arquitectura da Universidade de Lisboa, Portugal.

\section{SECRETARÍA TÉCNICA}

Dra. Gloria Rivero Lamela. Escuela Técnica Superior de Arquitectura. Universidad de Sevilla. España.

\section{EDITOR DEL NÚMERO Y COORDINACIÓN DE LOS CONTENIDOS CIENTÍFICOS}

Dr. Miguel Ángel de la Cova Morillo-Velarde. Escuela Técnica Superior de Arquitectura. Universidad de Sevilla. España.

\section{COMITÉ CIÉNTIFICO}

Dr. Carlo Azteni. DICAAR. Dipartimento di Ingegneria Civile, Ambientale e Architettura. University Of Cagliari. Italia.

Dra. Maristella Casciato. GETTY Research Institute, GETTY, Los Angeles. Estados Unidos.

Dra. Anne Marie Châtelet. École Nationale Supérieure D'Architecture de Strasbourg (ENSAS). Francia.

Dr. Jean Louis Cohen. Institute of Fine Arts, New York University. Estados Unidos.

Dra. Josefina González Cubero. Escuela Técnica Superior de Arquitectura. Universidad de Valladolid. España.

Dr. José Manuel López Peláez. Escuela Técnica Superior de Arquitectura. Universidad Politécnica de Madrid. España.

Dra. Maite Méndez Baiges. Departamento de Historia del Arte. Universidad de Málaga. España.

Dr. Dietrich C. Neumann. Brown University In Providence, Ri (John Nicholas Brown Center For Public Humanities And Cultural Heritage). Estados Unidos.

Dr. Víctor Pérez Escolano. Catedrático Historia, Teoría y Composición Arquitectónicas. Escuela Técnica Superior de Arquitectura. Universidad de Sevilla. España.

Dr. Jorge Torres Cueco. Catedrático Proyectos Arquitectónicos. Escuela Técnica Superior de Arquitectura. Universitat Politècnica de València. España.

Dr. ir. Frank van der Hoeven, TU DELFT. Architecture and the Built Environment, Netherlands

\section{CORRESPONSALES}

Pablo de Sola Montiel. The Berlage Centre for Advanced Studies in Architecture and Urban Design. Paises Bajos.

Dr. Plácido González Martínez. Tongji University Caup (College Of architectura \& Urban Planing). Shangai, China.

Patrícia Marins Farias. Faculdade de Arquitetura. Universidade Federal da Bahia. Brasil.

Dr. Daniel Movilla Vega. Umeå School of Architecture. Umeå University. Suecia.

Dr. Pablo Sendra Fernández. The Bartlett School of Planning. University College London. Inglaterra.

Alba Zarza Arribas. Centro de Estudos Arnaldo Araújo, Porto. Portugal.

Dra. María Elena Torres Pérez. Facultad de Arquitectura. Universidad Autónoma de Yucatán, Mérida. México.

\section{TEXTOS VIVOS}

Juan José López de la Cruz. Escuela Técnica Superior de Arquitectura. Universidad de Sevilla. España.

Dr. Francisco Javier Montero Fernández. Escuela Técnica Superior de Arquitectura. Universidad de Sevilla. España. Dra. Esther Mayoral Campa. Escuela Técnica Superior de Arquitectura. Universidad de Sevilla. España. 


\section{SERVICIOS DE INFORMACIÓN}

\section{CALIDAD EDITORIAL}

La Editorial Universidad de Sevilla cumple los criterios establecidos por la Comisión Nacional Evaluadora de la Actividad Investigadora para que lo publicado por el mismo sea reconocido como "de impacto" (Ministerio de Ciencia e Innovación, Resolución 18939 de 11 de noviembre de 2008 de la Presidencia de la CNEAI, Apéndice I, BOE n² 282, de 22.11.08).

La Editorial Universidad de Sevilla forma parte de la U.N.E. (Unión de Editoriales Universitarias Españolas) ajustándose al sistema de control de calidad que garantiza el prestigio e internacionalidad de sus publicaciones.

PUBLICATION QUALITY

The Editorial Universidad de Sevilla fulfils the criteria established by the National Commission for the Evaluation of Research Activity (CNEAI) so that its publications are recognised as "of impact" (Ministry of Science and Innovation, Resolution 18939 of 11 November 2008 on the Presidency of the CNEAl, Appendix I, BOE No 282, of 22.11.08).

The Editorial Universidad de Sevilla operates a quality control system which ensures the prestige and international nature of its publications, and is a member of the U.N.E. (Unión de Editoriales Universitarias Españolas-Union of Spanish University Publishers).

Los contenidos de la revista PROYECTO, PROGRESO, ARQUITECTURA aparecen en:

bases de datos: indexación
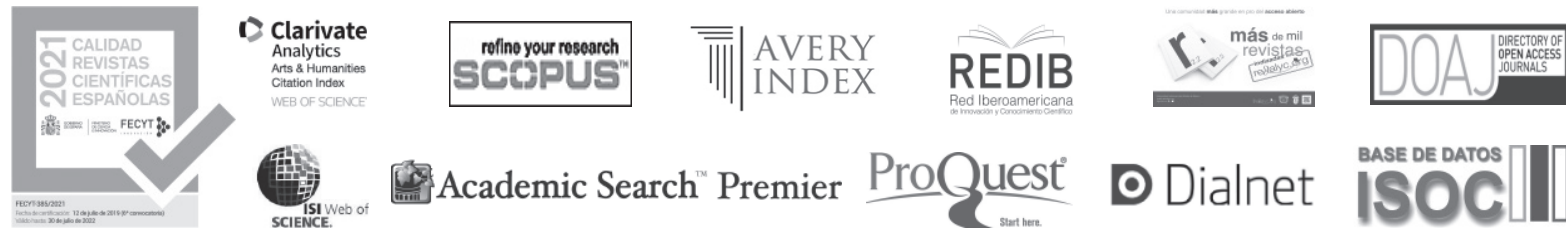

D Dialnet

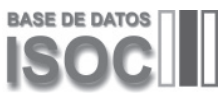

SELLO DE CALIDAD EDITORIAL FECYT 2019, 2020, 2021. Cuartil C2.

WoS. Arts \& Humanities Citation Index. JCl: 0,58. Cuartil Q2. Architecture

SCOPUS: SJR (2019): 0.11, H index: 2. Cuartil Q4

AVERY. Avery Index to Architectural Periodicals

REBID. Red Iberoamericana de Innovación y Conocimiento Científico. Cuartil Q2.

EBSCO. Fuente Académica Premier

EBSCO. Art Source

DOAJ, Directory of Open Access Journals

PROQUEST (Arts \& Humanities, full text)

DIALNET

ISOC (Producida por el CCHS del CSIC)

DRIJ. Directory of Research Journals Indexing

\section{catalogaciones: criterios de calidad}

RESH (Revistas Españolas de Ciencias Sociales y Humanidades).

Catálogos CNEAl (16 criterios de 19). ANECA (18 criterios de 21). LATINDEX (35 criterios sobre 36).

DICE (CCHS del CSIC, ANECA).

MIAR, Matriu d'Informació per a l'Avaluació de Revistes. IDCS 2018: 10,500. Campo ARQUITECTURA

CLASIFICACIÓN INTEGRADA DE REVISTAS CIENTÍFICAS (CIRC-CSIC): A

ERIHPLUS

SCIRUS, for Scientific Information.

ULRICH'S WEB, Global Serials Directory.

ACTUALIDAD IBEROAMERICANA.

\section{catálogos on-line bibliotecas notables de arquitectura:}

CLIO. Catálogo on-line. Columbia University. New York HOLLIS. Catálogo on-line. Harvard University. Cambridge. MA

SBD. Sistema Bibliotecario e Documentale. Instituto Universitario di Architettura di Venezia OPAC. Servizi Bibliotecari di Ateneo. Biblioteca Centrale. Politecnico di Milano

COPAC. Catálogo colectivo (Reino Unido)

SUDOC. Catálogo colectivo (Francia)

ZBD. Catálogo colectivo (Alemania)

REBIUN. Catálogo colectivo (España)

OCLC. WorldCat (Mundial) 
EVALUACIÓN EXTERNA POR PARES Y ANÓNIMA.

El Consejo Editorial remitirá el artículo a dos expertos revisores anónimos dentro del campo específico de investigación y crítica de arquitectura, según el modelo doble ciego.

El director de la revista comunicará a los autores el resultado motivado de la evaluación por correo electrónico, en la dirección que éstos hayan utilizado para enviar el artículo. El director comunicará al autor principal el resultado de la revisión (publicación sin cambios; publicación con correcciones menores; publicación con correcciones importantes; no aconsejable para su publicación), así como las observaciones y comentarios de los revisores.

Si el manuscrito ha sido aceptado con modificaciones, los autores deberán reenviar una nueva versión del artículo, atendiendo a las demandas y sugerencias de los evaluadores externos. Los artículos con correcciones importantes serán remitidos al Consejo Asesor para verificar la validez de las modificaciones efectuadas por el autor. Los autores pueden aportar también una carta al Consejo Editorial en la que indicarán el contenido de las modificaciones del artículo. Los artículos con correcciones importantes serán remitidos al Consejo Asesor para verificar la validez de las modificaciones efectuadas por el autor.

\section{DECLARACIÓN ÉTICA SOBRE PUBLICACIÓN Y MALAS PRÁCTICAS}

La revista PROYECTO, PROGRESO, ARQUITECTURA (PPA) está comprometida con la comunidad académica en garantizar la ética y calidad de los artículos publicados. Nuestra revista tiene como referencia el Código de Conducta y Buenas Prácticas que, para editores de revistas científicas, define el COMITÉ DE ÉTICA DE PUBLICACIONES (COPE).

Así nuestra revista garantiza la adecuada respuesta a las necesidades de los lectores y autores, asegurando la calidad de lo publicado, protegiendo y respetando el contenido de los artículos y la integridad de los mismo. El Consejo Editorial se compromete a publicar las correcciones, aclaraciones, retracciones y disculpas cuando sea preciso.

En cumplimiento de estas buenas prácticas, la revista PPA tiene publicado el sistema de arbitraje que sigue para la selección de artículos así como los criterios de evaluación que deben aplicar los evaluadores externos -anónimos y por pares, ajenos al Consejo Editorial-. La revista PPA mantiene actualizados estos criterios, basados exclusivamente en la relevancia científica del artículo, originalidad, claridad y pertinencia del trabajo presentado.

Nuestra revista garantiza en todo momento la condifencialidad del proceso de evaluación: el anonimato de los evaluadores y de los autores; el contenido evaluado; los informes razonados emitidos por los evaluadores y cualquier otra comunicación emitida por los consejos Editorial, Asesor y Científico si así procediese.

Igualmente quedan afectados de la máxima confidencialidad las posibles aclaraciones, reclamaciones o quejas que un autor desee remitir a los comités de la revista o a los evaluadores del artículo.

La revista PROYECTO, PROGRESO, ARQUITECTURA (PPA) declara su compromiso por el respeto e integridad de los trabajos ya publicados. Por esta razón, el plagio está estrictamente prohibido y los textos que se identifiquen como plagio o su contenido sea fraudulento, serán eliminados o no publicados por la revista PPA. La revista actuará en estos casos con la mayor celeridad posible. Al aceptar los términos y acuerdos expresados por nuestra revista, los autores han de garantizar que el artículo y los materiales asociados a él son originales o no infringen derechos de autor. También los autores tienen que justificar que, en caso de una autoría compartida, hubo un consenso pleno de todos los autores afectados y que no ha sido presentado ni publicado con anterioridad en otro medio de difusión.

\section{EXTERNAL ANONYMOUS PEER REVIEW.}

Editorial Board will be sent to two anonymous experts, within the specific field of architectural investigation and critique, for a double blind review.

The Director of the journal will communicate the result of the reviewers evaluations to the authors by electronic mail, to the address used to send the article. The Director will communicate the result of the review (publication without changes; publication with minor corrections; publication with significant corrections; its publication is not advisable), as well as the observations and comments of the reviewers, to the main author.

If the manuscript has been accepted with modifications, the authors will have to resubmit a new version of the article, addressing the requirements and suggestions of the external reviewers. The articles with corrections will be sent to Advisory Board for verification of the validity of the modifications made by the author. The authors can also send a letter to the Editorial Board, in which they will indicate the content of the modifications of the article.

\section{ETHICS STATEMENT ON PUBLICATION AND BAD PRACTICES} PROYECTO, PROGRESO ARQUITECTURA (PPA) makes a commitment to the academic community by ensuring the ethics and quality of its published articles. As a benchmark, our journal uses the Code of Conduct and Good Practices which, for scientific journals, is defined for editors by the PUBLICATION ETHICS COMMITTEE (COPE).

Our journal thereby guarantees an appropriate response to the needs of readers and authors, ensuring the quality of the published work, protecting and respecting the content and integrity of the articles. The Editorial Board will publish corrections, clarifications, retractions and apologies when necessary.

In compliance with these best practices, PPA has published the arbitration system that is followed for the selection of articles as well as the evaluation criteria to be applied by the anonymous, external peer-reviewers. PPA keeps these criteria current, based solely on the scientific importance, the originality, clarity and relevance of the presented article.

Our journal guarantees the confidentiality of the evaluation process at all times: the anonymity of the reviewers and authors; the reviewed content; the reasoned report issued by the reviewers and any other communication issued by the editorial, advisory and scientific boards as required.

Equally, the strictest confidentiality applies to possible clarifications, claims or complaints that an author may wish to refer to the journal's committees or the article reviewers.

PROYECTO, PROGRESO ARQUITECTURA (PPA) declares its commitment to the respect and integrity of work already published. For this reason, plagiarism is strictly prohibited and texts that are identified as being plagiarized, or having fraudulent content, will be eliminated or not published in PPA. The journal will act as quickly as possible in such cases. In accepting the terms and conditions expressed by our journal, authors must guarantee that the article and the materials associated with it are original and do not infringe copyright. The authors will also have to warrant that, in the case of joint authorship, there has been full consensus of all authors concerned and that the article has not been submitted to, or previously published in, any other media. 


\section{emergencias del espacio común}

índice

editorial

¿CÓMO VIVIREMOS TODOS JUNTOS? MENSAJES DESDE EL INTERIOR DE LA ARQUITECTURA /

HOW WILL WE LIVE ALL TOGETHER? MESSAGES FROM INSIDE ARCHITECTURE

Miguel Ángel de la Cova Morillo-Velarde - (DOl: http://dx.doi.org/10.12795/ppa.2021.i25.09)

artículos

LA INVENCIÓN DEL DESIERTO. ARTE Y ARQUITECTURA DESDE LA COSTA PERUANA / INVENTION OF THE DESERT. ART AND ARCHITECTURE FROM THE PERUVIAN COAST

Octavio Montestruque Bisso; Richard Asto Altamirano - (DOl: http://dx.doi.org/10.12795/ppa.2021.i25.01)

PROYECTO RIIS: UN HAPPENING PARA LA VIDA SOCIAL /

RIIS PROJECT: A HAPPENING FOR SOCIAL LIFE

Juan José Tuset Davó - (D0l: http://dx.doi.org/10.12795/ppa.2021.i25.02)

PLAYGROUNDS Y ESPACIO COMÚN. A PROPÓSITO DEL JUEGO EN LA CIUDAD SUSPENDIDA / PLAYGROUNDS AND COMMON SPACE. ON PLAYING IN THE SUSPENDED CITY

José Parra-Martínez; Nicolás Stutzin-Donoso; Juan Manuel López-Carreño - (DOl: http://dx.doi.org/10.12795/ppa.2021.i25.03)

LA CALLE COMO ESCENOGRAFÍA URBANA. LAS TRANSFORMACIONES DE LARIOS Y ALCAZABILLA EN EL SIGLO XX / THE STREET AS URBAN SCENOGRAPHY. THE TRANSFORMATIONS OF CALLE LARIOS AND CALLE ALCAZABILLA IN THE TWENTIETH CENTURY

Luisa Alarcón González; Francisco Javier Montero-Fernández - (D0l: http://dx.doi.org/10.12795/ppa.2021.i25.04)

DEL BRICOLAJE AL TALLER O EL ESPACIO DE LA TRANSFORMACIÓN EN FRANCIA /

FROM DO-IT-YOURSELF TO THE WORKSHOP, OR THE TRANSFORMATION SPACE IN FRANCE

Ophelia Mantz - (DOI: http://dx.doi.org/10.12795/ppa.2021.i25.05)

reseña bibliográfica TEXTOS VIVOS

IÑAKI ÁBALOS: PALACIOS COMUNALES ATEMPORALES. GENEALOGÍA Y ANATOMÍA

Daniel Díez Martínez - (DOl: http://dx.doi.org/10.12795/ppa.2021.i25.06)

LE CORBUSIER: ¿CAÑONES, MUNICIONES? IGRACIAS! VIVIENDAS... POR FAVOR JORGE TORRES CUECO Y JUAN CALATRAVA: UNA EXPOSICIÓN, UN PABELLÓN Y UN LIBRO: LE CORBUSIER, 1937-1938

Ricardo Sánchez Lampreave - (DOI: http://dx.doi.org/10.12795/ppa.2020.i25.07)

VICTORIANO SAINZ GUTIÉRREZ: ALDO ROSSI Y SEVILLA. EL SIGNIFICADO DE UNOS VIAJES

José lgnacio Linazasoro Rodríguez - (DOl: http://dx.doi.org/10.12795/ppa.2021.i25.08) 


\title{
DEL BRICOLAJE AL TALLER O EL ESPACIO DE LA TRANSFORMACIÓN EN FRANCIA
}

\author{
FROM DO-IT-YOURSELF TO THE WORKSHOP, OR THE TRANSFORMATION \\ SPACE IN FRANCE
}

Ophelia Mantz (https://orcid.org/0000-0003-2243-3088)

RESUMEN En el contexto pandémico actual, Francia, como tantos otros países, quiere impulsar nuevos modos de habitar el espacio doméstico post-COVID. Sin embargo, ciertos modelos parecen surgidos de un proceso más lento de transformación. Veamos cómo el bricolaje -más allá de una simple actividad marginal- podría simbolizar cambios paradigmáticos en la sociedad francesa. Por ello, se presentan las características y la evolución del bricolaje en Francia gracias al impulso de las ciencias sociales en los años setenta. Se explica cómo el bricolaje es capaz de generar un cambio de comportamiento ciudadano y con ello ofrecer un nuevo modelo económico y productivo. Sin embargo, para poder participar tanto en la vivienda del siglo XXI como en la ciudad contemporánea, la acción sobre la materia debe ser legitimada espacialmente. Por ello, se presenta el papel histórico del Homo faber, junto con su taller, en la construcción de la cité. Finalmente, se establece un paralelismo entre la revalorización del bricolaje y el papel del grand bricoleur, ya desempeñado por nuevas generaciones de arquitectos franceses.

El bricolaje como "ser en el mundo" para la construcción de un nuevo modelo económico y productivo en una etapa de transición ecológica.

PALABRAS CLAVE taller; bricolaje; unidad productiva; transición ecológica; homo faber

ABSTRACT Within the context of the current pandemic, France, like many other countries, is hoping to promote new ways of inhabiting domestic space after COVID. However, certain models seem to have emerged from a slower process of transformation. Herein we will examine how do-it-yourself work (DIY), beyond just a marginal activity, may be indicative of paradigm changes in French society. To achieve this, the essay describes the characteristics and changes in DIY across time in France based on the momentum created by the social sciences in the 1970s. It explains how DIY is able to generate changes in the people's behavior and thus offer the possibility of a new model for the economy and production. However, in order to be able to play a role in both twenty-first century housing and the contemporary city, action taken on matter must be spatially legitimized. Therefore, we also discuss the historical role of homo faber, along with his workshop, in the construction of la cité. Last of all, parallels are drawn between the renewed appreciation of DIY and the role of the grand bricoleur, or large-scale DIY practitioner, already played by the latest generations of French architects.

Thus, DIY is viewed as a way of "being in the world" that will lead to the construction of a new economic and productive model during an era of ecological transition.

KEYWORDS workshop; do-it-yourself; productive unit; ecological transition; homo faber

Persona de contacto / Corresponding author: omantz@uh.edu. University of Houston. Estados Unidos. 
1. Untitled (portable planetarium) 2009 Obra de Sarah Sze (1969, Boston) La Sucrière Xe Biennale de Lyon "Le spectacle du quotidien" Commissaire: Hou Hanru. dalbera from Paris, France.

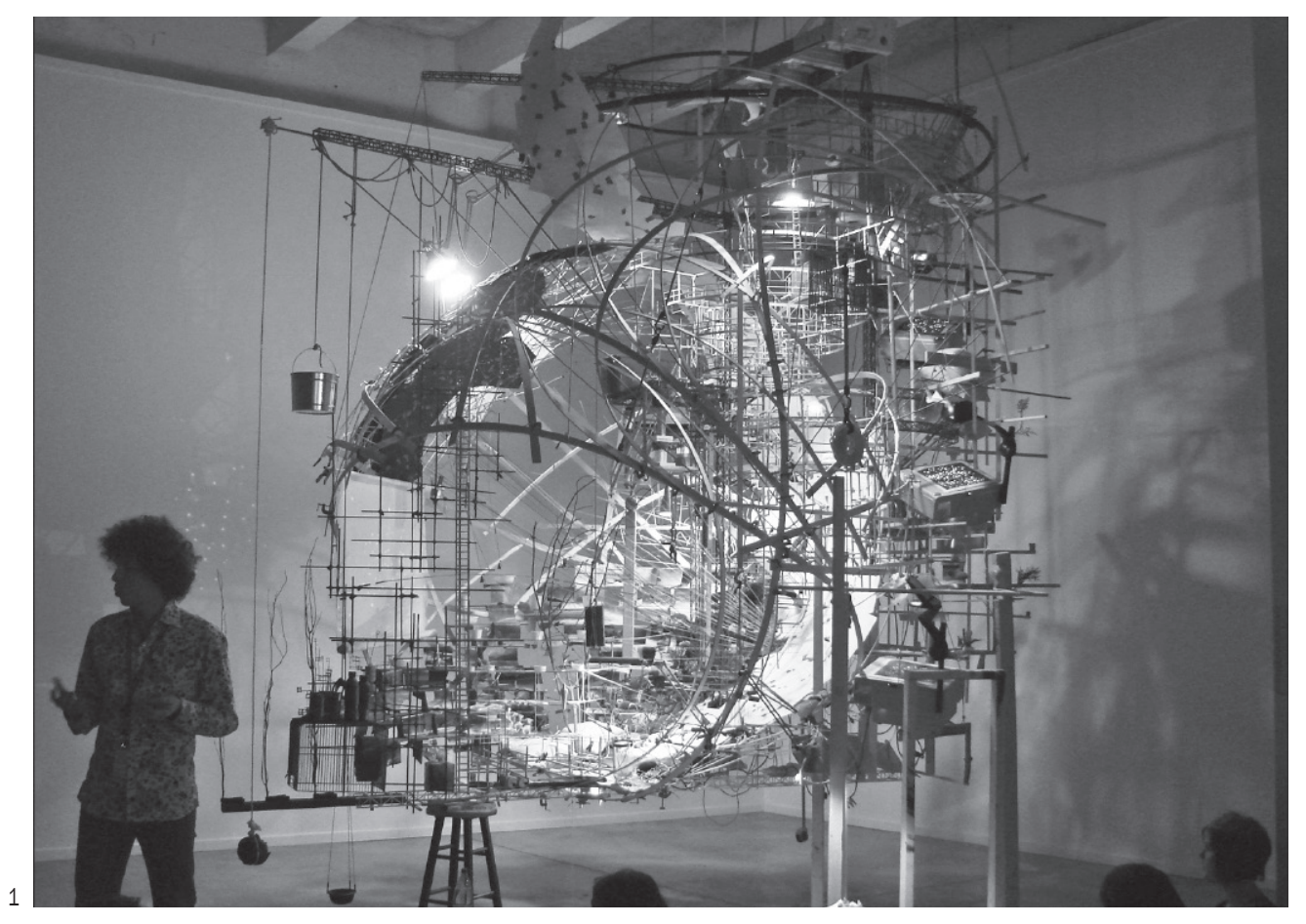

\section{INTRODUCCIÓN}

E I 17 de marzo de 2020 a las 12 h, Francia decreta su primer confinamiento oficial en respuesta a la dimensión pandémica de la COVID-19. Con anterioridad a la prohibición de desplazamientos dentro del país, París, capital de la nación y modelo de una política territorial históricamente centralizada, se vacía. Las poblaciones de parisinos con segunda residencia, estudiantes o jóvenes trabajadores, ya expertos en supervivencia en los espacios mínimos que ofrece la capital, no dudan en reconquistar un mundo rural menos saturado. Diez días más tarde, el 27 de marzo, Jean-Michel Normand, periodista del diario Le Monde, analiza la práctica del bricolaje en tiempos de pandemia. El artículo traducido como "Hacer bricolaje en tiempos de confinamiento o la sociedad del consuelo"1, analiza cómo la venta y el uso de las herramientas de bricolaje han ido reorganizando la nueva cotidianidad de los ciudadanos franceses.

Antes de la crisis sanitaria de la COVID-19, la actividad manual ya estaba en pleno crecimiento. Esta había empezado a desarrollarse en los sectores, no solo populares, sino de la burguesía media. El sociólogo Ronan Chastellier enlaza este frenesí por el bricolaje (figura 1) con el crecimiento de una consciencia colectiva por el re-uso y el reciclaje de los objetos que conforman nuestros espacios domésticos. El abuso de la obsolescencia programada de los objetos cotidianos parece haber promovido, en la sociedad francesa, cierta actitud contra el consumo y, con ello, un impulso de la creatividad.

El hombre contemporáneo está condicionado por varios vectores de contingencia que han acelerado su forma de habitar. Las crisis climático-sanitarias han aumentado el sentimiento de abstracción y de impotencia. A su vez, la pandemia nos ha impuesto límites espaciales drásticos con un horizonte temporal difuso. En este contexto, quizá el bricolaje, descrito por Hugues Bazin² como proceso existencial y contingente, podría impulsarnos hacia un nuevo modelo de progreso. Una vía de desarrollo donde el hombre vuelve a equiparse con herramientas manuales y con una mayor proximidad en el

1. NORMAND, Jean-Michel. Bricoler en temps de confinement ou la société de consolation. En: Le Monde, 27 de marzo de 2020.

2. BAZIN, Hugues. L'art de Bricolage. Bricoleurs d'art. En: Les Cahiers d'Artes. L'art à l'épreuve du social. Bordeaux: Presses Universitaires de Bordeaux, 2013, n. ${ }^{\circ}$, p. 2. 
proceso técnico-productivo. Entonces, el bricolaje podría ser una oportunidad para recobrar confianza en el poder de acción del ciudadano.

La interpretación de este momento podría estar sujeta a diferentes puntos de vista o marcos territoriales. Sin embargo, sería interesante analizar la actualidad de la crisis sanitaria dentro de un territorio y de una cultura específica: Francia, país que ha mantenido en sus costumbres la práctica del bricolaje. A lo largo de este texto se intentará transmitir y articular cómo acontecimientos socioculturales e históricos particulares de la cultura francesa han podido desencadenar el crecimiento actual del bricolaje al que se ha referido Chastellier. En esta actividad, considerada por muchos como un espacio de resistencia, se puede vislumbrar un impulso para perseguir nuevos modos de habitar el espacio doméstico; cómo "ser en el mundo" o être-au monde.

En este ensayo se analizan, en primer lugar, las características y la evolución de las actividades relacionadas con el bricolaje. Con ello, se hará hincapié en el potencial de acción de esta actividad en relación con el proceso de individuación del hombre. En segundo lugar, se puede vislumbrar una posible participación del bricolaje en el cambio de modelo económico y productivo actual hacia un sistema más sostenible, conocido como transición ecológica. En la tercera parte, se estudia el papel histórico del espacio del taller como construcción de tejido social y urbano en Francia. Finalmente, se establecerá un paralelismo entre la revalorización del bricolaje y la redefinición del perfil del arquitecto francés contemporáneo, heredero de los profundos cambios curriculares que fueron impulsados en los años setenta.

La hipótesis del artículo reside en demostrar que las prácticas emergentes en el campo de la arquitectura francesa podrían derivar también de un mundo que re-ensambla lo existente, y le da valor a lo obsoleto. En lo contemporáneo, el bricolaje parece imponerse como herramienta y medio de cambio para la construcción de un nuevo paradigma. Con ello, se alumbrará la necesidad de dotar de vida y recuperar el taller como espacio de lo común en una nueva dimensión de la vivienda del siglo XXI.

\section{EL BRICOLAJE: ORIGEN Y EVOLUCIÓN DE UNA ACTIVIDAD MARGINAL}

El término francés bricolage deriva de la palabra italiana briccola utilizada en la Edad Media hacia 1360 para designar una catapulta. El origen etimológico de la palabra está también en la raíz del término francés bricoles, que se usa para designar un conjunto de objetos residuales o accesorios sin mucho valor. Más allá de esta connotación, a veces peyorativa, bricolaje comúnmente tiende a referirse a una actividad de autoproducción que refleja una relación privilegiada del habitante con los objetos ${ }^{3}$.

En el siglo XX, la etnología, la sociología y la antropología 4 francesa han estado marcadas por el estudio de las actividades de lo cotidiano. El bricolaje, como expresión de técnicas polivalentes frente a la materia y objetos residuales, aparece ligado al espacio doméstico. En esta actividad, a veces marginada, muchos investigadores han valorado el proceso de individuación que conlleva. Aquí el término individuación se refiere a la construcción psicológica del individuo que procura articular el sujeto corporal y la sociedad. Desde los años noventa, gracias a la cultura anglosajona del do it yourself (DIY), el bricolaje ha sido identificado tanto como un potencial empresarial como una actividad resiliente ${ }^{5}$. Dentro del marco cultural francés, esta actividad, ciertamente marginal, ha sido centro de interés desde hace varias décadas. Desde Michel de Certeau ${ }^{6}$ a Georges Perec ${ }^{7}$, el bricolaje simboliza

3. CALVEZ, Marcel. Le bricolage dans l'aménagement de la maison. Approche à partir de 20 pavillons en lotissements HLM. París: Ministère de la Culture, 1987. Artículo que reúne los resultados de una investigación financiada por la "Mission du Patrimoine Ethnologique".

4. WARNIER, Jean-Pierre. Construire la culture matérielle: L’homme qui pensait avec ses doigts. París: Presses Universitaires de France, 1999. El autor, etnólogo, propone una amplia bibliografía desde Marcel Mauss hasta Michel de Certeau, Bourdieu, Baudrillard y Latour.

5. DUYMEDJIAN, Raffi. La France snobe les vertus du bricolage. En: Le Monde, 2 de abril de 2013. Hace referencia a Karl Weick, profesor de psicología y gestión en La Ross School of Business de la Universidad de Michigan, USA.

6. CERTEAU, Michel de. L'invention du quotidien. T. 1 y 2. [1. ${ }^{\text {a }}$ ed., 1980] París: Gallimard, 1990.

7. PEREC, Georges. L'Infra-ordinaire. París: Seuil, 1996. Col. "Librairies du XXe siècle”. 
un espacio de resistencia frente a la lógica de producción de la sociedad de consumo.

Quizá pueda residir en este recinto de la contingencia el camino hacia un modelo de sociedad descrito por Ivan Illich como es el de la convivencialidad ${ }^{8}$. Illich se refiere a una estructura social en la que se aseguran los derechos a utilizar la energía para trabajar y vivir de manera creativa. El bricolaje inspira, interroga sobre las relaciones del hombre con los objetos cotidianos. Sus premisas residen en la indeterminación, la incertidumbre, la contingencia, el azar y el caos; todos ellos lo alzan como símbolo del proceso en sí, sin proyecto específico. El bricolaje se percibe como el medio para alcanzar la autonomía creadora; rechaza la separación entre búsqueda y acción, actividad manual e intelectual, creación y producción. En otras palabras, gracias a un acercamiento puramente empírico, el hombre del ensamblaje, liberado de la subordinación, deja de proyectar una visión hegemónica sobre la realidad, para inventar una nueva manera de mirarla.

En su libro La pensée sauvage, el antropólogo estructuralista Claude Lévi-Strauss establece una relación analógica entre la ciencia primitiva y el bricolaje. Lo define como técnica de lo concreto. En el primer capítulo titulado "La ciencia de lo concreto" recuerda cómo en el Neolítico se conforma la práctica del hombre en las grandes artes de la civilización como la cerámica, el telar, la agricultura o la domesticación de animales. Todas estas técnicas son el resultado de siglos de observación activa y metódica ${ }^{9}$, fruto de una experiencia repetitiva y de perseverancia. El bricolaje está descrito como una actividad manual cuyo resultado construye un conocimiento de lo concreto. Su práctica consiste en ensamblar elementos heteróclitos, residuales, previamente clasificados por el bricoleur. Esta actividad revela la capacidad de derivar el uso original de los objetos que re-ensambla y manipula. Dicho de otra manera, el hombre del bricolaje busca, sin prejuicios establecidos, ofrecer un nuevo orden y cohesión en un repertorio de objetos, de herramientas e informaciones que él mismo se ha dado; ofrece una solución basada en un conjunto de conocimientos polivalentes sobre los lugares de intervención, los conocimientos de los objetos y los materiales que los componen. Una de las características del bricolaje es la recuperación de materiales en desuso que, previamente a cualquier proyecto, se agrupan y conforman una nueva fuente de materia prima disponible. Detrás de esta actividad, ciertamente autodidacta, el hombre se complace en manejar la economía de los medios, agudizar su capacidad de invención y actuar como único actor del proceso constructivo.

En otro comentario, Lévi-Strauss habla de la dimensión mítico-poética del bricolaje ${ }^{10}$, estableciendo analogías con el castillo del cartero (facteur) Cheval o con los decorados de Georges Méliès (figura 2). Todos los objetos que conforman el inventario del bricoleur, hombre del bricolaje, se convierten en un tesoro que puede dar nacimiento a un mundo estético de gran valor. El bricolaje está constituido de residuos de obras humanas, considerados como un sous-ensemble o subcultura $^{11}$. El antropólogo define el bricolaje como la elaboración de estructuras generadas por el ensamblaje de residuos de acontecimientos, trozos, pedazos, testigos de la historia de un individuo o de una sociedad $^{12}$. Lévi-Strauss establece una relación entre el pensamiento mítico o mágico y el bricolaje. Recuerda que el artista nace del encuentro entre el sabio y el mundo del bricolaje ${ }^{13}$.

El bricolaje nos interesa en tanto que revela o recuerda al hombre su capacidad de encontrar soluciones a problemas concretos. Un valor caracterizado en el artesano tal y como lo describe Richard Sennett ${ }^{14}$ en su libro

8. ILLICH, Ivan. Tools for Conviviality. New York: Harper \& Row, 1973.

9. LÉVI-STRAUSS, Claude. La pensée sauvage. París: Plon, 1962, p. 20.

10. Ibíd., p. 29.

11. Ibíd., p. 31.

12. Ibíd., p. 34.

13. Ibíd., p. 35.

14. SENNETT, Richard. The Craftsman. Londres: Yale University Press, 2008. 


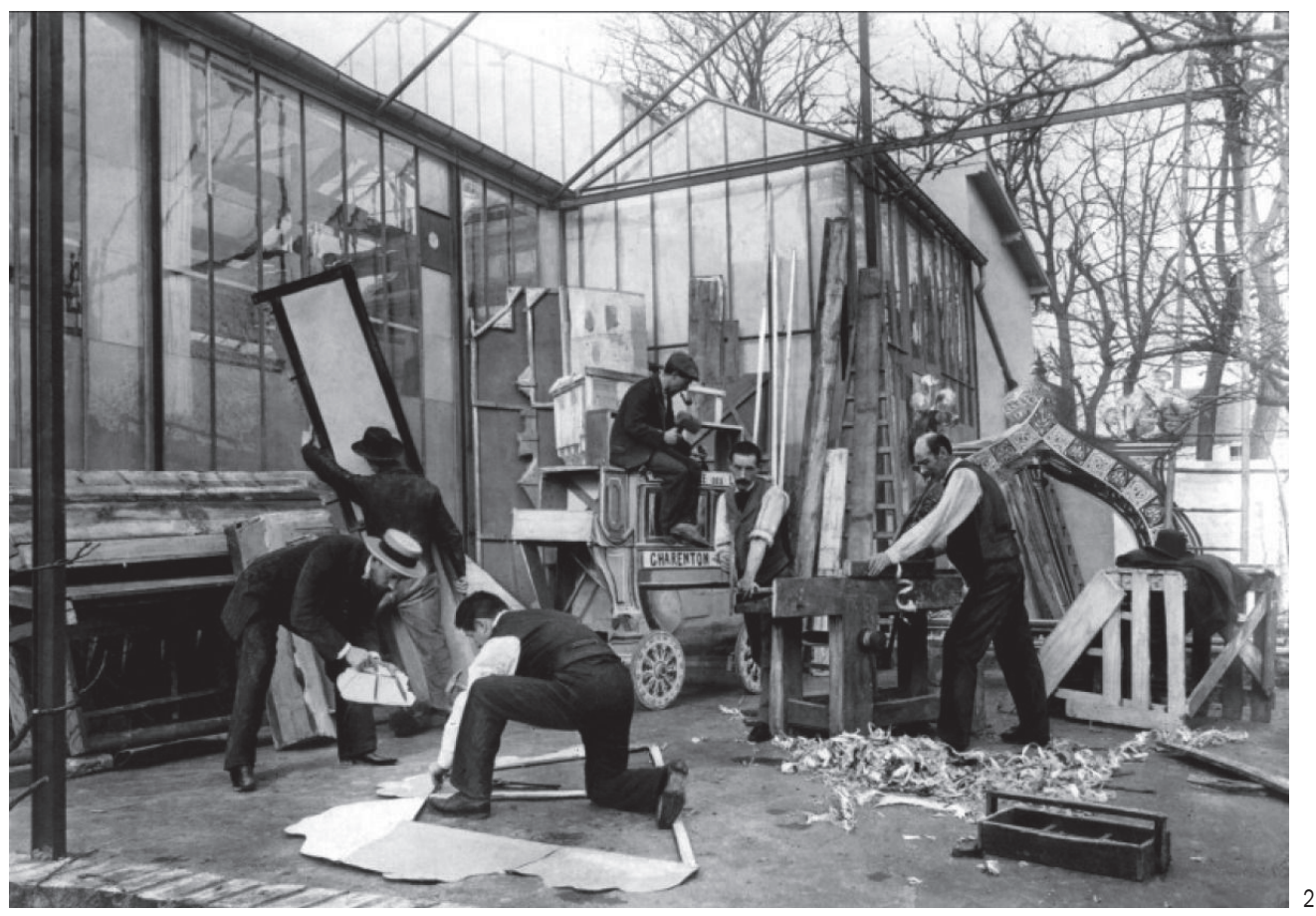

The Craftsman. El re-uso y el ensamblaje de objetos desde la economía de materiales residuales aportan un valor cualitativo desde un conocimiento técnico polivalente. Nos interesa ver en esta actividad, no profesional, cómo el hombre adquiere técnicas corporales que revelan su capacidad de acción sobre los objetos. Su cuerpo se compromete con la cultura material, definida por el etnólogo J. P. Warnier y el sociólogo J. F. Bayart como un vehículo de subjetivación en la medida en que contribuye a la constitución de sujetos morales y a la producción de relaciones sociales ${ }^{15}$. En otras palabras, la relación física del hombre con la materia participa en su proceso de individuación subjetiva y colectiva ${ }^{16}$.

Según Hervé Sika ${ }^{17}$, la actividad del bricolaje reside en devolver al arte una dimensión relacional. También recuerda que la realidad del hombre del bricolaje es heterogénea, des-jerarquizada y liberadora. Entonces, el bricolaje pone al sujeto en relación con los objetos residuales. Los transforma a través de unas técnicas corporales $^{18}$ adquiridas desde la experiencia o aprendidas en otros escenarios. Estas técnicas del cuerpo permiten categorizar y revalorar la materia original. El cuerpo pien$\mathrm{sa}^{19}$ y puede significar el valor de la materia a través de su gesto ${ }^{20}$ en un sistema de connotaciones y comunicación. En otras palabras, el cuerpo es materia que trabaja y que es trabajada ${ }^{21}$, produce y es producto.

\section{EL BRICOLAJE: HERRAMIENTA DE TRANSFORMACIÓN HACIA UNA TRANSICIÓN ECOLÓGICA}

En el contexto pandémico actual, la "acción" sobre la materia, representada en la escala doméstica del bricolaje, aparece como una oportunidad de cambio hacia una transición ecológica. En este sentido, el bricolaje agudiza la necesidad de saber enfrentarnos a la contin-

15. WARNIER, Jean-Pierre; BAYART, Jean-Francois. Matière à politique. Le pouvoir, les corps et les choses. París: Karthala, 2004, p. 319. Col. "Recherches internationals".

16. JULIEN, Marie-Pierre; ROSSELIN, Céline. La culture matérielle. París: La Découverte, 2005, p. 65.

17. SIKA, Hervé. Conférence gesticulée et dansée. Be bricoleur Baby. Extracto de la conferencia, texto dictalografiado, 2012.

18. MAUSS, Marcel. Les techniques du corps, sociologie et anthropologie. París: PUF, 1950 [1936]. Artículo originalmente publicado en el Journal de Psychologie, XXXII, 15 marzo-15 abril 1936, n. 3-4. Comunicación presentada a la Société de Psychologie el 17 de mayo de 1934.

19. WARNIER, Jean-Pierre, op. cit. supra, nota 4.

20. BERTHOZ, Alain. Le sens du mouvement. París: Odile Jacob, 1977. El autor estudió cómo el gesto de los trabajadores de la asociación caritativa EMMAÜS, instigada por l'Abbé Pierre (obispo francés, defensor de los pobres), va re-cualificando los residuos de ropa y objetos. Pone de manifiesto la importancia del movimiento y de la gestualidad de los trabajadores dentro del proceso de selección que permite recalificar la materia en desuso.

21. JULIEN, Marie-Pierre; ROSSELIN, Céline, op. cit. supra, nota 16. 
gencia y nos ofrece la oportunidad de ser actores de un proceso productivo manual ${ }^{22}$. Siguiendo los términos de Richard Sennett, se podría considerar la actividad del bricolaje como la emergencia de nuevas formas hacia acciones colectivas ${ }^{23}$. La acción por delante de la consciencia.

Quizá esta actividad sea también una oportunidad para destronar la hegemonía "del ojo", de lo visual como sentido supremo ${ }^{24}$ que nos aliena. Con el fin de profundizar en la idea de que "la acción en la materia" es una etapa decisiva en la evolución del hombre, utilizaremos el argumento del etnólogo Jean-Pierre Warnier basado en "pensar con los dedos". Una postura también defendida por arquitectos como Juhani Pallasmaa en su libro The Thinking Hand en 2009 o Flores y Prats en Pensado a mano.

En su libro, Construire la culture matérielle: L'homme qui pensait avec ses doigts ${ }^{25}$, J. P. Warnier explica cómo las técnicas corporales y el compromiso dinámico en la cultura material se articulan con las representaciones del mundo que dan identidad al hombre. El autor describe el proceso de hominización desde la relación establecida con la materia. Utiliza la idea de hominización culturobípeda del primatólogo Toshisada Nishida. Este pionero japonés, gracias a sus estudios sobre los chimpancés, destacó la importancia del comportamiento cultural en los animales que da lugar a la apertura hacia un nuevo "nicho ecológico". La constitución de un nicho ecológico significa elegir un medio específico, así como los elementos que permitan la vida y la reproducción de la especie según nuevas modalidades. En otras palabras, la constitución de un nicho ecológico se establece desde la aceptación de nuevas reglas en el juego de la existen$\mathrm{cia}^{26}$. Warnier defiende que la herramienta, dentro de la evolución del hombre, es fruto de una decisión motora operada en el enfrentamiento entre sociedad, cultura y medio $27, y$, por tanto, según Nishida, participaría en la definición de un nuevo nicho ecológico. Por estas razones, se propone ver en la actividad del bricolaje la posibilidad de establecer un nicho que impulse nuevas reglas, nuevos valores y nuevos comportamientos hacia un modelo de desarrollo más sostenible. Este sería el resultado de la construcción de un nuevo medio de existencia para el hombre en correspondencia con el bricolaje como herramienta de acción y medio para ser en el mundo.

Con el fin de aportar una reflexión sobre las limitaciones tipológicas del espacio doméstico contemporáneo en Francia, y quizá en otros países, este artículo propone considerar la posibilidad de "reintroducir" el espacio del bricolaje, o espacio de ensamblaje de materias residuales, en el programa doméstico. Con objeto de dar profundidad histórica a la cuestión, se estudia y analiza, a continuación, el papel del espacio del taller en Francia anterior a la revolución industrial. Este análisis permite explorar la posibilidad de reintegrar un espacio que parte de la vivienda para ser un elemento generador de tejido urbano: del taller a la cité.

\section{HOMO FABER O CÓMO CONSTRUIR \\ LA CITÉ DESDE EL TALLER}

Antes de ser atribuido exclusivamente al artista, el taller o atelier ha navegado entre diferentes términos como boutique o échoppe (figura 3) que significan: el espacio de trabajo del artesano. En cualquier caso, estos términos, siempre han estado cargados de connotaciones comerciales. En un caso u otro, estos espacios siempre han representado la dimensión espacial de la elaboración de objetos. Es allí donde el hombre, artesano o artista, ha ensamblado y transformado materias y/o objetos. Este devenir de la materia de estos objetos se apoya fundamentalmente en el desarrollo de técnicas corporales. Tal

22. ARENDT, Hannah. The Human Condition. University of Chicago Press, 1958, p. 285.

23. SENNETT, Richard. The Open City. En: HAAS, Tigran; WESTLUND, Hans, eds. In The Post-Urban World. Londres: Routledge, 2017. Presentación en la Universidad de Harvard para el Graduate School of Design en 2013.

24. JAMESON, Fredric. Le postmodernisme ou la logique culturelle du capitalisme tardif. París: Beaux-Arts de Paris Editions, 2007, p. 42. Texto original inglés: Postmodernism, or, the Cultural Logic of Late Capitalism. Duke University Press, 1991.

25. WARNIER, Jean-Pierre, op. cit. supra, nota 4.

26. Ibíd., p. 44.

27. Ibíd., p. 47. 


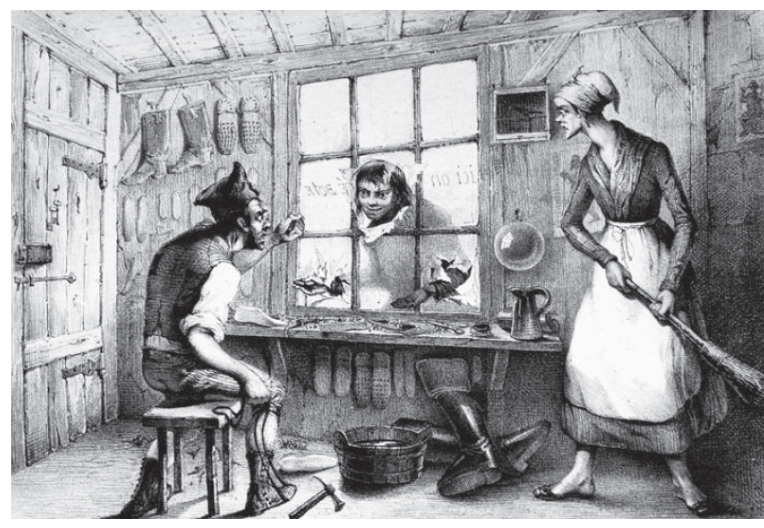

3

y como hemos mencionado anteriormente, en el espacio del taller, el cuerpo del hombre se pone en acción sobre la materia y es capaz de aportar un nuevo valor a la materia residual. Marcel Mauss, padre de la etnología francesa, definía estas técnicas corporales como los modos en que los hombres transmitían, de sociedad a sociedad y de manera tradicional, el cómo servirse de su cuerpo. El espacio del taller, relacionado directamente o indirectamente con el espacio doméstico, permitía alzar las cualidades creativas de un hombre llamado Homo faber ${ }^{28}$. El urbanista Richard Sennett recuerda que el hombre que fabrica es respetado en la ciudad mediante una práctica modesta. El Homo faber tiene una ética de producción que implica una relación específica con la cité. La primacía de la cité surge con el hacer derivado del habitar ${ }^{29}$. El Homo faber instiga un comportamiento, una ética de la que nace la idea de cité. Para Sennett, el hacer es la cité mientras que la ville es el habitar. Pero en el siglo XX la cité y la ville se distancian. Con ello se desintegra el Homo faber, el actor social de la ciudad.
3. Representación pintoresca de un taller o échoppe de zapatero. Litografía, 1838.

4 y 5 . Planche 1: localización de los talleres en los pueblos de casas agrupadas, ejemplo de SaintAndré de Rosans entre 1739 y 1836. Planche 2: localización de talleres-tiendas en pueblos de casas dispersas. Comparación entre 1599 y 1836.

Hasta la revolución industrial, el hombre que fabrica herramientas es el actor principal de la organización social. Sin embargo, a pesar de la llegada de la máquina -y con ella la sectorización del trabajo en el proceso constructivo-, el desarrollo del modelo económico industrial del siglo XIX no consiguió borrar de un plumazo la existencia del taller. Su proceso de desaparición fue mucho más lento y progresivo. Estas características que le son propias al carácter resiliente del bricoleur refuerzan la idea de que el espacio de transformación material pueda ser un espacio de resistencia ante la lógica productiva. El taller, símbolo del mundo artesanal, perseveró y fue un espacio estructurante como unidad tipológica del habitar extendida a la ocupación territorial. Este espacio se impuso como vector de organización de mayor escala en el hábitat rural, así como en el tejido urbano ${ }^{30}$ (figuras 4 y 5). El taller, independiente o ligado al espacio doméstico, fue un actor en la organización social y económica francesa hasta finales del siglo XIX.

Cabe recordar que en la transición de la prehistoria a la historia, el manejo técnico de materiales como la piedra, el bronce, la arcilla, etc., genera un cuerpo artesanal a partir del cual se establecen diferentes modelos de sociedades $^{31}$. Por esta razón, se entiende que el taller es la representación espacial del mundo artesanal. En el siglo XIX, el Diccionario razonado de la arquitectura francesa del siglo XI al XVI, del arquitecto Eugène Viollet-le-Duc, consta de varias descripciones de boutique o tienda, como tipologías de espacios domésticos. En ciudades tan importantes como París o en las estructuras urbanas de menos población que dibujan el mundo rural, el taller, o boutique, es parte integrante de la arquitectura en el territorio $^{32}$ (figuras 6 y 7). El taller, íntimamente ligado al espacio doméstico, se individualiza como espacio de trabajo a lo largo de la historia. Gracias a su presencia en fachada, el espacio-taller consigue expresarse arquitectónicamente

28. BOURG, Dominique; PAPAUX, Alain. Dictionnaire de la pensée écologique. París: PUF, 2015, pp. 536-540.

29. SENNETT, Richard. Construir y habitar. Ética para la ciudad. Barcelona: Anagrama, 2019, pp. 14-18.

30. BELMONT, Alain. Les “boutiques" des artisans villageois dans la France d'Ancien Régime (XVle-début du XIXe siècle). En: Annales de Bretagne et des pays de l'Ouest, 1999, t. 106, n. ${ }^{\circ}$, pp. 197-210.

31. BRAUDEL, Fernand. L'identité de la France. Les hommes et les choses. París: Arthaud, 1986.

32. BELMONT, Alain, op. cit. supra, nota 30. 


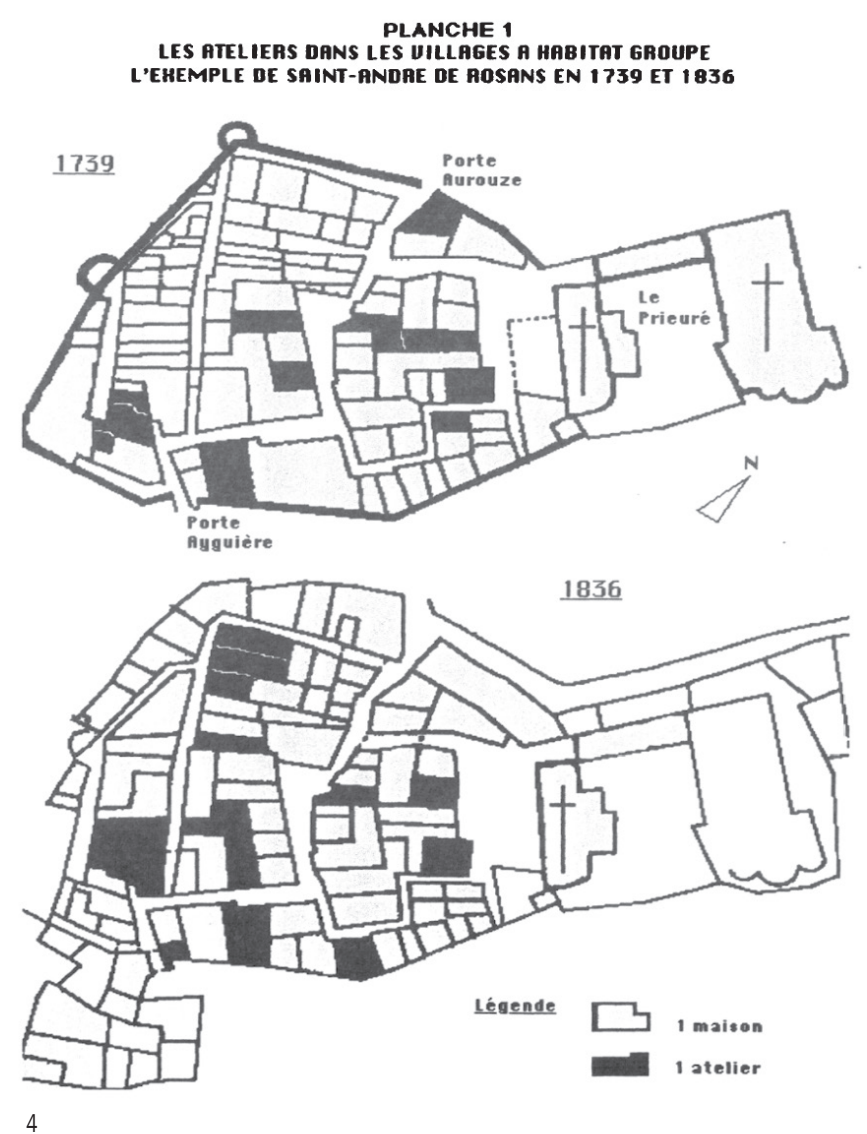

y, por tanto, legitimarse en el espacio público de la calle. Con ello, se convierte en un elemento fundamental en la construcción de los centros urbanos en Francia. En otras palabras, esa transformación, que reveló la profesionalización del artesano, impulsó al taller-boutique como actor del medio urbano ${ }^{33}$.

J.-M. Gourden en su libro Le peuple des ateliers. Les Artisans du XIX siècle (El pueblo de los talleres. Los Artesanos del siglo XIX) describe cómo, antes y después de la revolución industrial, el taller representaba la organización económica y social francesa. El taller se mantiene como elemento de la fábrica urbana casi hasta principios del siglo XX. Pero la llegada del progreso técnico de la máquina de vapor -y con ella el proletariado- desestabiliza al actor del taller. El artesano vive el proceso de industrialización como una desposesión ${ }^{34}$. Su lenta agonía arrastra

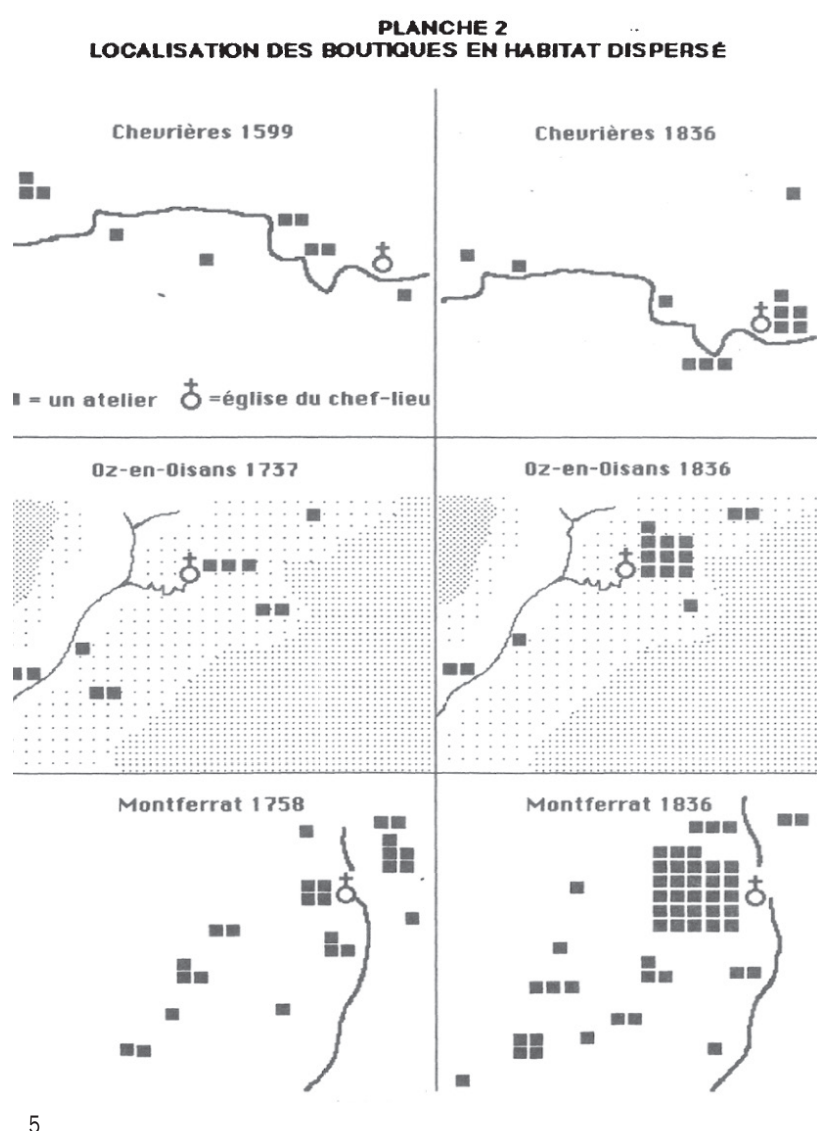

progresivamente la desaparición del taller y su disolución a favor de la fábrica se llevará a cabo durante casi un siglo entero. Pero esta lentitud también demuestra que, más allá de una actividad manual, el taller ha sido un espacio de resistencia.

Según la filósofa Hannah Arendt, la imposición del modelo industrial convirtió al artesano, también descrito como Homo faber, en un animal laborans ${ }^{35}$. Una definición que apunta a una transformación negativa y deshumanizante del hombre del siglo XIX. El hombre, históricamente protagonista de la fabricación de herramientas, se transforma en un esclavo del proceso de producción impuesto por la industrialización. El taller o unidad productiva, donde el hombre realiza su proceso de individuación subjetiva y social a través de sus técnicas corporales e instrumentales sobre la materia,

33. Ídem.

34. GOURDEN, Jean-Michel. Le peuple des ateliers. Les Artisans du xix siècle. París: Creaphis, 1992.

35. ARENDT, Hannah, op. cit. supra, nota 22, pp. 327-337. 
6. Alzado y Planta Baja. Casa en Châteaudun, France. Principios del siglo XIV, B representa la boutique.

7. Plantas y Alzado, Casa Ciudad o Maison de Ville de la ciudad de Cluny, Francia, siglo XII. Planta baja A: la boutique en D, la galería o pórtico abierto en $\mathrm{E}$, el patio en $\mathrm{F}$, la cocina en $\mathrm{H}$ con su gran chimenea I. Un pozo está en $\mathrm{G}$. La primera planta, trazada en B, con la escalera en $\mathrm{K}$, la sala en $\mathrm{L}$, la galería abierta $\mathrm{o}$ con vidrios en $\mathrm{N}$ con una pequeña escalera para acceder al bajo cubierta, y un dormitorio en 0 .

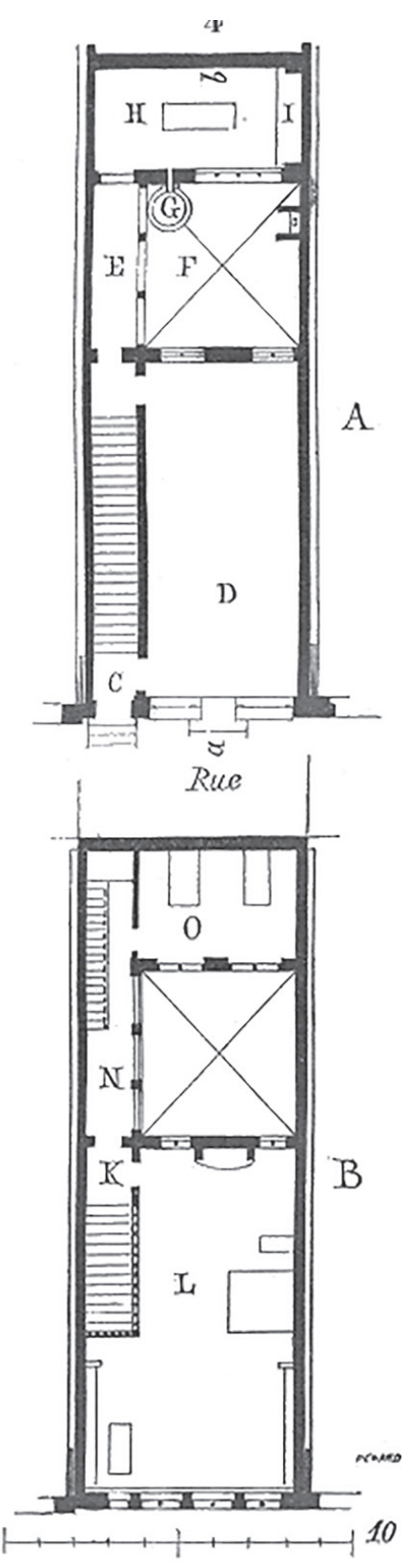

20

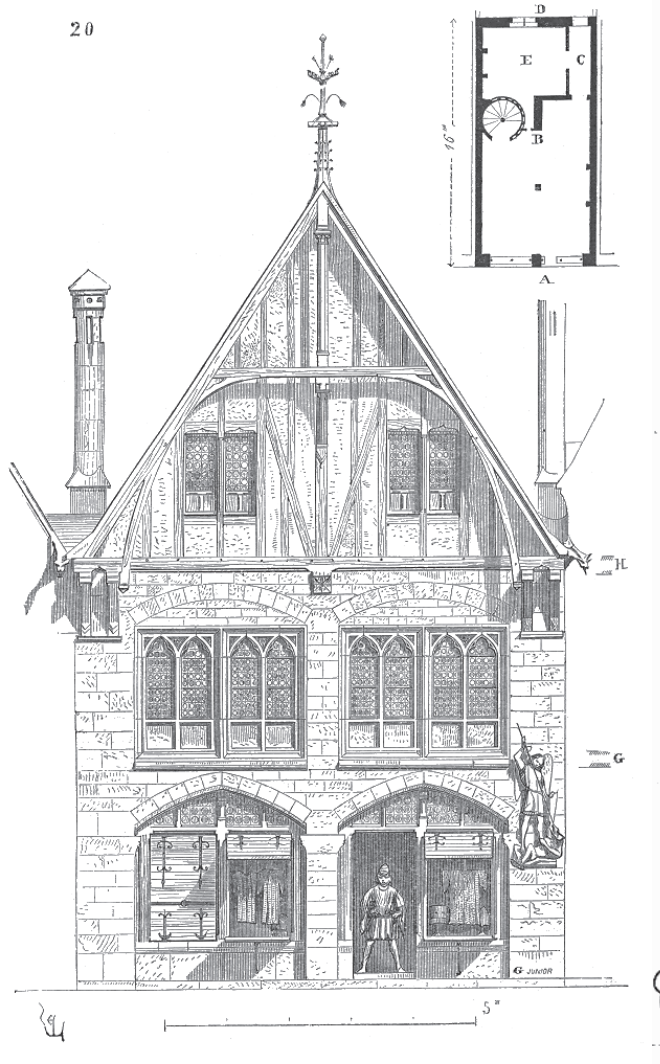

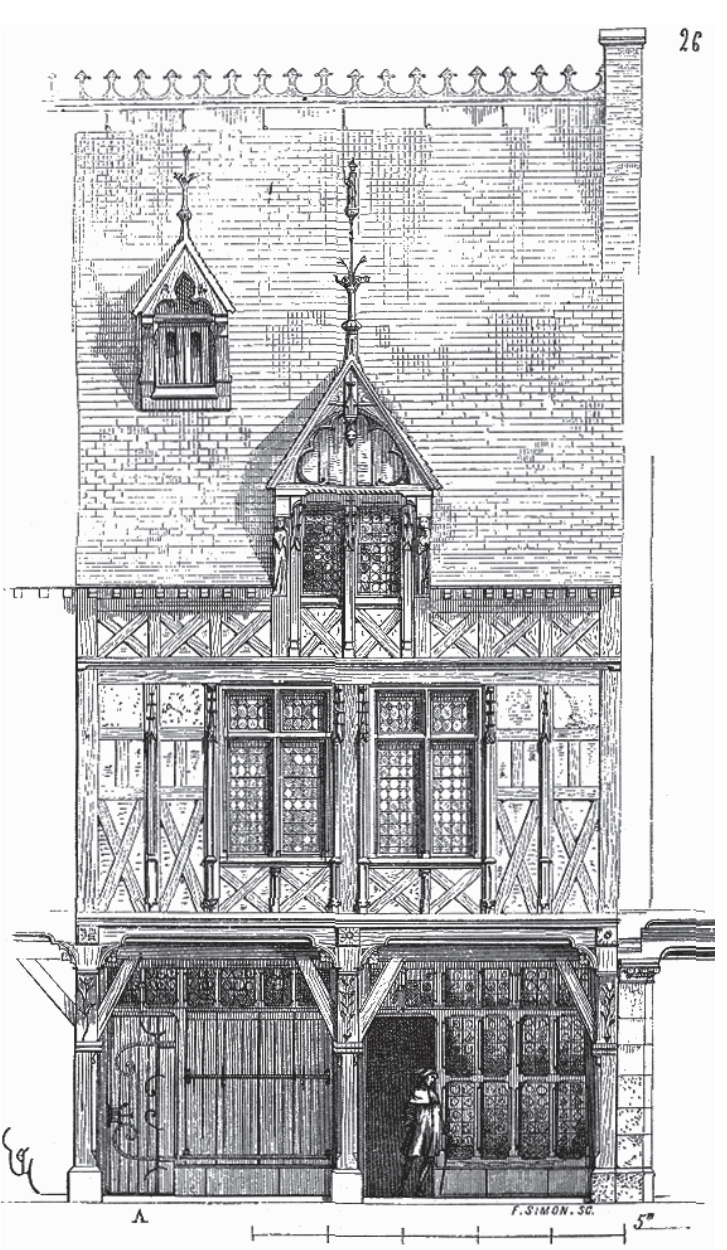

7 
desaparece a lo largo del siglo XIX. El término boutique o tienda se desplaza al entorno de las artes mecánicas. Como consecuencia, es le chantier o la obra que define el espacio del obrero. El espacio del taller sobrevive solo y exclusivamente para satisfacer la existencia del artista ${ }^{36}$. Esta mutación programática sucumbe con la publicación de La Enciclopedia -o Dictionnaire raisonné des sciences, des arts et des métiers, de Denis Diderot (1713-1784) y de Jean Le Rond d'Alembert (1717-1783)-, que legitima para siempre su propósito exclusivamente artístico. El diccionario que símboliza el apetito de conocimiento del siglo XVIII en Francia, el siglo de las luces o siècle des lumières, formaliza una redefinición profunda de la sociedad como de sus formas materiales ${ }^{37}$.

Tal y como hemos visto previamente, los siglos XIX y XX borran el espacio del taller dentro de la fábrica urbana en Francia y contemplan la desaparición del Homo faber como actor de la cité ${ }^{38}$. Sin embargo, la actividad del bricolaje se mantiene en el espacio doméstico. El bricolaje, considerado como actividad indefinida y marginal, se convierte en estudio de trabajo por parte de las ciencias humanas y sociales en la segunda mitad del siglo XX.

El futuro de un espacio de la transformación o bricolaje converge con el elogio de Guillaume Logé -en el Diccionario del pensamiento ecológico ${ }^{39}$ - en la integración creadora como modelo económico capaz de satisfacer al hombre en su necesidad de sentido ${ }^{40}$. El autor menciona al economista Jeremy Rifkin ${ }^{41}$, defensor en el año 2011 de la posibilidad de una tercera revolución industrial. El economista-ecologista americano con su postura teórica establece modelos económicos sostenibles basados en la innovación y en la creación de valor. Estos modelos darían lugar a lo que él llama un capitalismo cultural. Este nuevo modelo económico más horizontal se ve impulsado por Internet, por la aparición de nuevas herramientas domésticas y por la reducción de la cadena del valor. Este nuevo marco de acción desencadenaría el desarrollo de una economía distribuida y cooperativa ${ }^{42}$. Un modelo que dará al hombre una mayor autonomía, en vez de tenerlo bajo control.

\section{EL GRAND BRICOLEUR: CAMBIO DE PARADIGMA PARA LA SOCIEDAD FRANCESA}

Sobre un periodo de cincuenta años, el desarrollo y el avance de la sociología, la antropología, la etnología y la filosofía han impactado la estructura curricular de las escuelas de arquitectura en Francia. En los años 196768, Francia vivió la revolución estudiantil Ilamada Mayo del 68 que impulsó grandes cambios en la sociedad francesa de posguerra. En este contexto, el ministro de Asuntos Culturales, André Malraux, decidió reformar la enseñanza en Francia. Max Querrien, con la ayuda de Henri Raymond, íntimo de Henri Lefebvre, decidieron introducir las ciencias humanas y sociales en los estudios de arquitectura ${ }^{43}$. Aquello cultivó un perfil de "arquitectosociólogo" que podría facilitar esta transición en el espacio de la vivienda.

36. GRIENER, Pascal. La notion d'atelier de l'Antiquité au XIXe siècle: chronique d'un appauvrissement sémantique. En: Perspective, 2014, n. ${ }^{\circ} 1$, p. 20. El autor nos recuerda que la Enciclopedia de Diderot y D'Alembert fue redefiniendo el taller como el espacio exclusivamente atribuido al artista, reservando al obrero una relación con el chantier o la obra; el espacio de la boutique o tienda se ve atribuido a las artes mecánicas.

37. RAGON, Michel. Histoire de l'architecture et de l'urbanisme modernes (3 t.). París: Seuil, 1991. T. 1. Idéologies et pionniers, 1800-1910. Col. "Point Essais". 38. SENNETT, Richard. Construir y habitar. Ética para la ciudad. Barcelona: Anagrama, 2019, pp. 14-18.

39. BOURG, Dominique; PAPAUX, Alain. Dictionnaire de la pensée écologique. París: PUF, 2015, pp. 61-64.

40. AUGÉ, Marc. Non-lieux. Introduction à une anthropologie de la surmodernité. París: Seuil, 1992.

41. RIFKIN, Jeremy. La Troisième Révolution industrielle: Comment le pouvoir latéral va transformer l'énergie, l'économie et le monde [The Third Industrial Revolution: How Lateral Power Is Transforming Energy, the Economy, and the World] (trad. del inglés). París: Les liens qui libèrent, 2012,380 p. El libro fue muy criticado en Francia por el filósofo Dominique Bourg y el político ecologista Noël Mamère.

42. LOGÉ, Guillaume. Art. En: Dictionnaire de la pensée écologique. BOURG, Dominique; PAPAUX, Alain, dirs. París: PUF, 2015, pp. 61-64.

43. CHADOIN, Olivier. Le sociologue chez les architectes, matériau pour une sociologie de la sociologie en situation ancillaire. En: Sociétés contemporaines. París: Presses de Sciences Po, 2009, p. 84. En 1968, una reforma de la escuela tradicional de Bellas Artes permite independizar los Ateliers d'Architecture que dieron nacimiento a las actuales ENSA (Escuelas Nacionales Superiores de Arquitectura). 
8 y 9. Casa Latapie construida en 1993 en Floirac, Francia. Arquitectos: Anne Lacaton y Jean-Philippe Vassal.

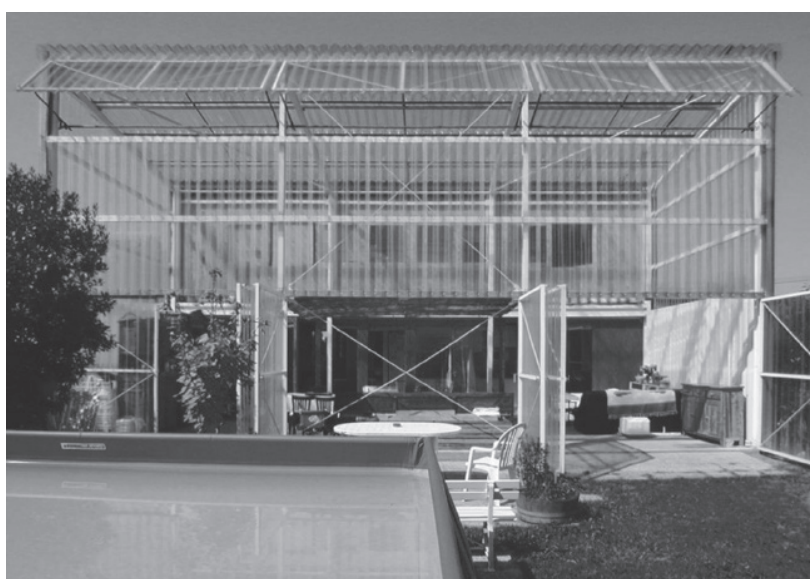

8

A pesar del impacto de las ciencias sociales en la secuencia curricular de las escuelas de arquitectura francesas, la producción tipológica del espacio doméstico ha visto tímidamente aparecer nuevas maneras de vivir en el siglo XXI. Según Monique Eleb -en lo relativo a la oferta tipológica de la vivienda ${ }^{44}$-, a pesar de que el poder de acción del arquitecto francés haya sido desplazado a las promotoras, nuevas generaciones de profesionales parecen preparadas y dispuestas a ofrecer nuevas maneras de pensar la vivienda del siglo XXI. Cabe destacar que la sensibilidad de los arquitectos formados desde una visión pluridisciplinar del espacio habitado permite ver nuevos modelos espaciales, así como prácticas profesionales que erigen el "hacer más espacio con menos medios". Quizá el contexto de la pandemia pueda estimular y legitimar las prácticas emergentes de una nueva generación de arquitectos franceses.

A finales del siglo XX, los arquitectos Anne Lacaton y Jean-Philippe Vassal han promovido un vector de cambio a través de diferentes proyectos de viviendas: hacer más con menos y hacer con lo que existe reduciendo las demoliciones. La casa Latapie construida en 1993 en Floirac, Francia, seguramente sea la más emblemática de las

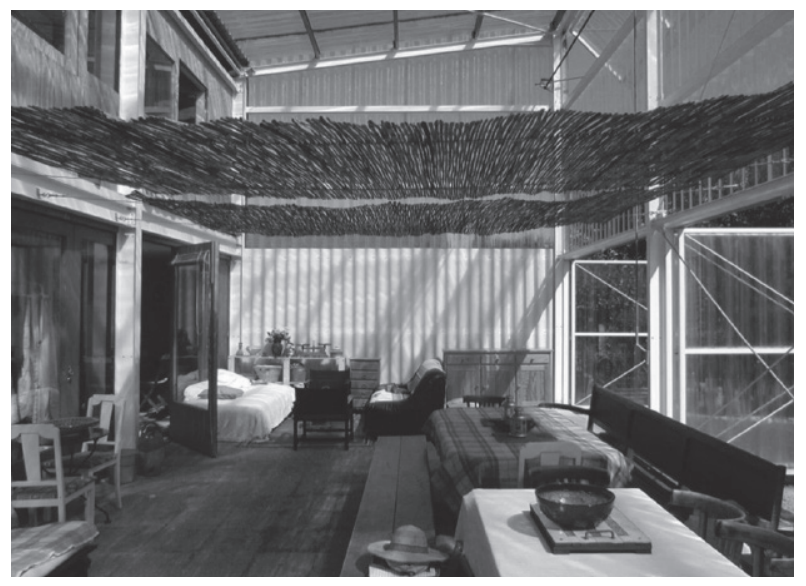

9

estrategias para la construcción de nuevos espacios domésticos (figuras 8 y 9). Sin duda, la ingeniosidad del uso de materiales prestados del mundo rural y agrícola, junto con la asunción de ofrecer un espacio desjerarquizado y heterogéneo, han permitido transgredir muchos códigos semánticos del espacio del hogar. Cabe destacar cómo la casa ha permitido revelar un espíritu autónomo y sin prejuicios. Isabelle Regnier, periodista en Le Monde, describe las obras de este tándem de arquitectos como cargadas de un cierto encanto "bricolado" 45 .

En paralelo a estos experimentos del "más con menos" inducido por Lacaton y Vassal, en Marsella, Francia, en 1992 nace un proyecto llamado La Friche la Belle de $\mathrm{Mai}^{46}$. Este proyecto, en continua transformación, ilustra el cambio en la forma de habitar y la construcción de la fábrica urbana. El proyecto se define como un experimento político, lugar de pensamiento y acción en torno a la redefinición de la relación entre arte, territorio y sociedad. El proyecto, heterogéneo y ciertamente informal, nació desde la reconquista de una antigua fábrica. Su génesis basada en la actividad artística de diferentes actores (artistas, residentes, intelectuales) sigue ciertas pautas de la actividad del bricolaje: anticipar sin fijar, construir con flexibilidad,

44. ELEB, Monique. Entre confort, désir et normes: le logement contemporain (1995-2010). Bruselas: Mardaga, 2013.

45. REGNIER, Isabelle. Le Pritzker d'architecture pour les francais Anne Lacaton et Jean-Philippe Vassal. En: Le Monde, 16 de marzo de 2021.

46. Disponible en: https://www.lafriche.org/. 
experimentar y potenciar el espíritu de cooperación. Varios arquitectos franceses han participado como Matthieu Poitevin y Pascal Reynaud o el estudio Agence ConstruireSébastien Eymard et Loïc Julienne, con Simon Himpens.

En el camino hacia el grand bricoleur, otro acontecimiento, más cerca de la actualidad, desembarcó en el pabellón francés de la Exposición Internacional de Arquitectura de 2018 en Venecia (Exposición comisariada por las arquitectas irlandesas Yvonne Farrell y Shelley McNamara). A priori, la instalación del pabellón no ha tenido mucha repercusión mediática, aunque aquí nos interesa re-contextualizar culturalmente lo que podría estar emergiendo: mientras el pabellón suizo elogiaba el vacío, el pabellón francés (figuras 10 y 11) liderado por el estudio Encore Heureux interpreta el tema del freespace ${ }^{47}$ desde una visión humanista. Los miembros del estudio de arquitectura parisino defendían el papel del arquitecto como impulsor de la transición ecológica.

El pabellón exponía una larga panoplia de objetos cotidianos. Al conservar, recuperar, reusar objetos, materiales, estructuras, etc., el arquitecto aparece como el actor que dispone, re-organiza lo existente. Se promueve el papel de un arquitecto que navega entre los objetos cotidianos y los ensambla. Se convierte en el hombre de un bricolaje de mayor escala, capaz de aportar valor a la materia disponible por encima de la carga semántica de los materiales manufacturados. En otros términos, a través de este ejemplo, lo que interesa es recobrar la idea original presentada al inicio de este artículo, en la que la acción corporal sobre la materia es generadora de valor cualitativo en el mundo material del hombre.

En línea con el cambio de paradigma en la sociedad francesa, la legitimidad de la vivienda participativa podría ser un acontecimiento relevante en la idea de autoproducción. En 2014, un nuevo concepto de vivienda,

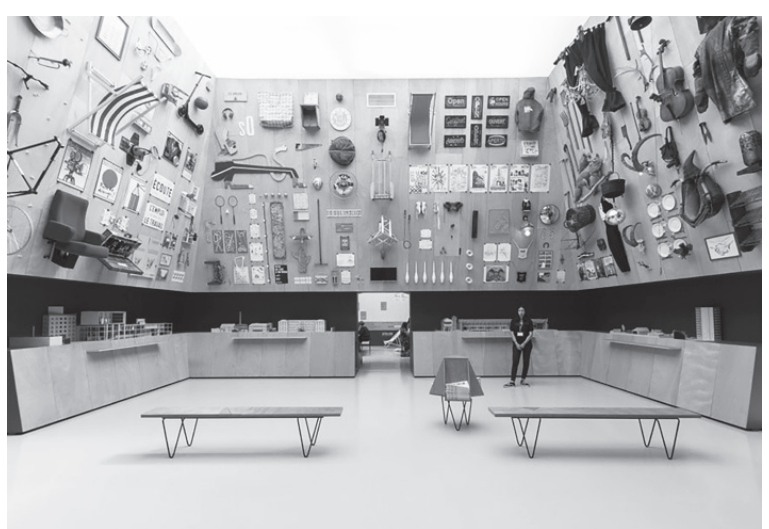

10

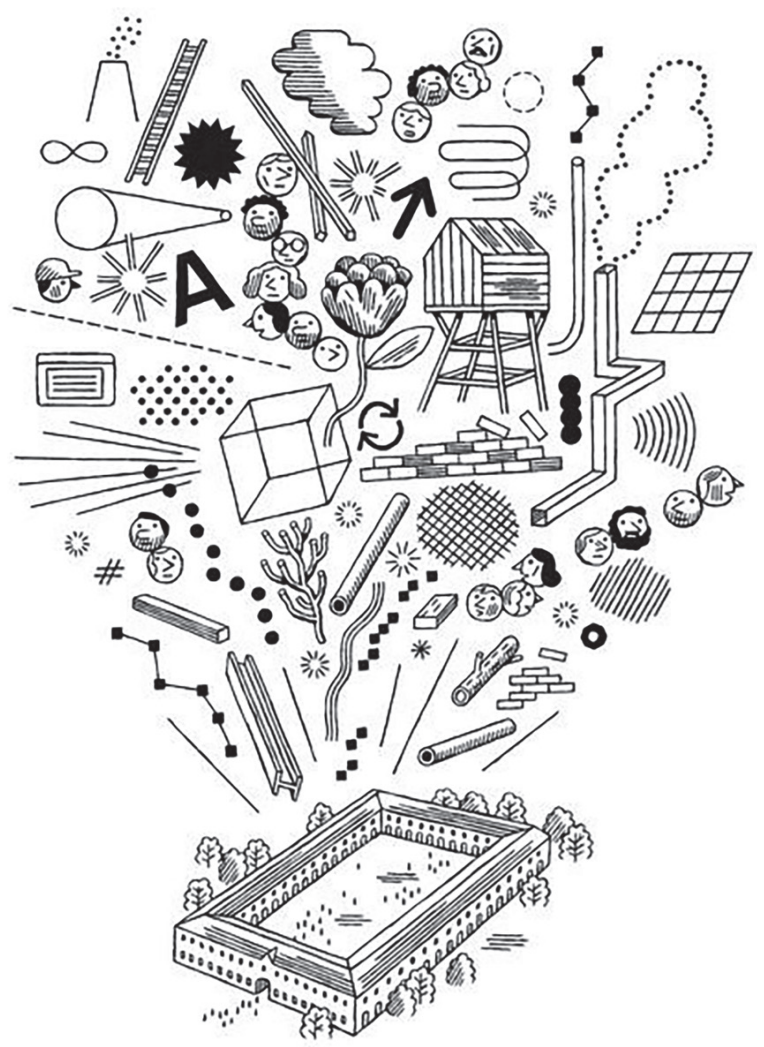

11

definida como participativa o logements participatifs ${ }^{48}$, fue legislado por una ley llamada ALUR. Este nuevo modelo, aunque todavía en vías de desarrollo, sigue los pasos de países como Alemania, Suiza o Noruega. En Francia, la

47. Bienal de Arquitectura de Venecia, del 26 de mayo al 25 de noviembre de 2018. Disponible en: www.labiennale.org/en.

48. La vivienda participativa se basa en un enfoque ciudadano: permite a grupos de personas construir sus viviendas y compartir una forma de vida ecológica y comunitaria, a un menor costo. Está recogida en la ley ALUR. El marco cooperativo tiene como objetivo cambiar la forma de vida y la organización de los edificios, hacer que los habitantes sean activos y con ello luchar contra la especulación.

La vivienda participativa se define, en esta ley, como un enfoque ciudadano que permite unir fuerzas entre personas físicas y personas jurídicas, para participar en la definición y diseño de sus viviendas e instalaciones. Todos los actores participan en las decisiones relacionadas con la construcción o adquisición del edificio y, en su caso, en la gestión de edificios. Disponible en: https://www.ecologie.gouv.fr/habitat-participatif-cadre-juridique-habiter-autrement. 


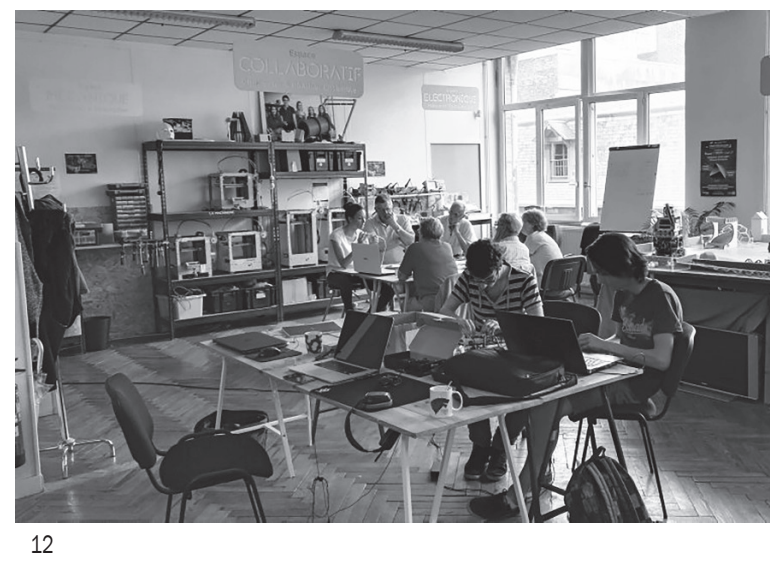

ley ALUR ha modificado el código de la construcción de viviendas. Esta normativa agrupa marcos legislativos y reglamentarios relativos a la construcción, la promoción inmobiliaria y las viviendas sociales en Francia.

Dicha ley marca la voluntad de ofrecer nuevas maneras de vivir en agrupamientos en tiempos de transición ecológica, anterior a la crisis sanitaria de la COVID-19. Gracias a la inercia de este impulso político, la unión social por el habitar, Fabrique de la ville et Transit City, presenta un informe en noviembre del 2020 en el que se propone institucionalizar el bricolaje ${ }^{49}$. El contexto de la pandemia parece haber acelerado proyectos ya en marcha. Quizá, los haya enfatizado. Y gracias a la crisis sanitaria, los políticos los han legitimado finalmente. Los espacios llamados fab lab (figura 12), living lab y otros makerspace se han impuesto en el territorio francés recibiendo oficialmente el 19 de septiembre del 2020 un desbloqueo financiero de 110 millones de euros por parte del Estado.

Del impulso de las ciencias sociales de los años de posguerra a la legitimación del espacio del bricolaje en plena pandemia, pasando por la emergencia de
Figuras 10 y 11. Pabellón francés en la Exposición Internacional de Arquitectura en Venecia, Italia, 2018. Estudio Encore Heureux.

12. Espacio fab lab de La Machinerie, en Amiens. 13. La Bricothèque.

14. Espacio de brico-working.

arquitectos-bricoleurs, todos apuntan hacia un cambio significativo en la sociedad francesa. Todos dejan claro la necesidad de reconocer otros modos de hacer y de habitar. En nuestro caso concreto, el hecho de querer institucionalizar el espacio del bricolaje -la bricoteca o bricothèque $^{50}$ (figuras 13 y 14) - es, sin duda, una etapa constituyente de un cambio de paradigma hacia la transición ecológica.

La recuperación del taller/estudio podría introducir una mayor flexibilidad programática en el espacio de la vivienda. Una neutralidad más consecuente con las contingencias de la vida. En definitiva, podríamos afirmar que el espacio doméstico tiende a ser considerado ya no tanto como una vivienda, sino como una unidad productiva.

El apoyo político en Francia, probablemente impulsado por la crisis sanitaria y ecológica, ha permitido revalorizar estos espacios de integración creativa que pasan de ser un fenómeno alternativo y marginal a un espacio vanguardista de resistencia y autoproducción.

\section{CONCLUSIONES}

Híbridos, reversibles y multiformes son las nuevas características de los espacios del habitar francés. Gracias a las nuevas herramientas miniaturizadas de la gran industria -impresora 3D, cortadora láser, equipos digitales, etc.el Homo faber parece haber renacido de sus cenizas. El hombre innovador, autónomo, y resiliente aparece como el resultado del sacrificio del animal laborans o proletariado de los siglos XIX y XX. El animal laborans ha renunciado a su individualidad para poder pertenecer a un cuerpo social desempeñado en el mundo de la fábrica. Sin embargo, la figura de resistencia, que caracteriza al Homo faber ${ }^{51}$, lo ha convertido en el actor de una transformación política más profunda encaminada hacia la transición ecológica. El nuevo espacio de la "bricoteca" o bricothèque parece poder navegar entre diferentes escalas: de lo doméstico al espacio público institucionalizado. En los dos

49. VELEZ, Véronique; DEMERY, Cécile; MERCURIO, Céline Di. Etude prospective: Nouveaux usages et modes d'habiter [en línea]. París: L’Union Sociale pour l'Habitat, 2020 [consulta: 07-10-2021]. Disponible en: https://www.union-habitat.org/centre-de-ressources/patrimoine-maitrise-d-ouvrage/nouveaux-usageset-modes-d-habiter-analyse-d.

50. Ibíd., p. 24

51. ARENDT, Hannah, op. cit. supra, nota 22, p. 332. 


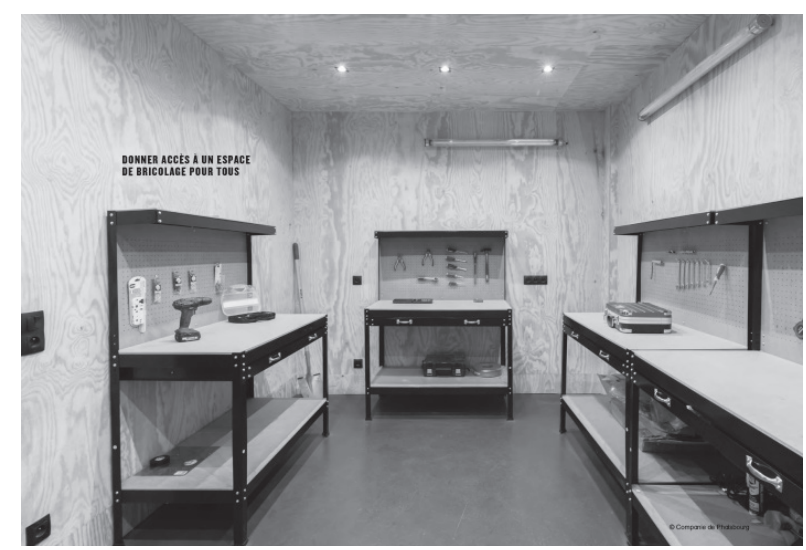

13

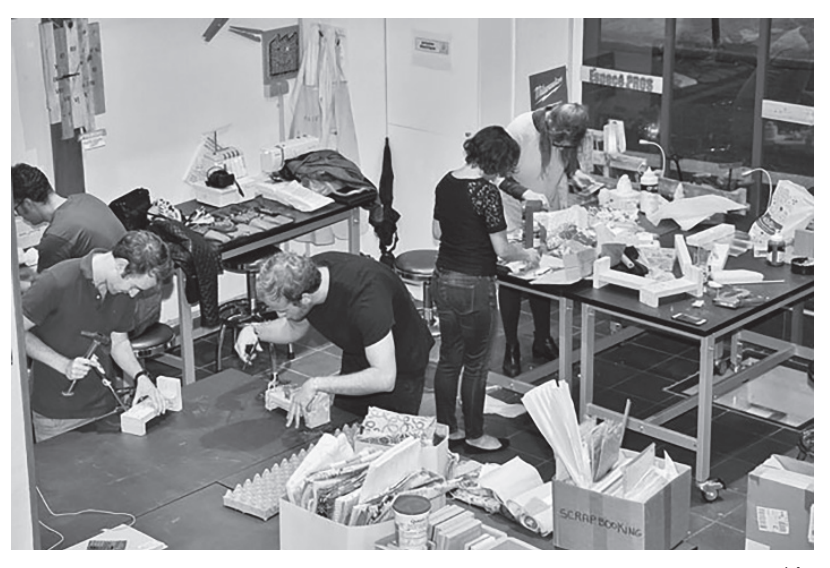

14 casos, el bricolaje, como actividad técnica polivalente originada desde las clases obreras, parece alumbrar un cambio profundo en la sociedad francesa: la construcción de un nuevo nicho ecológico.

El espacio común del taller ofrece la posibilidad de reintroducir el espacio de la transformación de la materia en la tipología de la vivienda del siglo XXI. Sin embargo, nos referimos a un espacio potencial, multiforme, informal y heterogéneo que privilegie el acto de transformar la materia y con ella su valor semántico. Este espacio parece cobrar sentido en el seno de la vida doméstica contemporánea arrastrada por la acumulación de objetos y flujos de materias residuales ${ }^{52}$. Pero más allá del taller la vivienda podría ser proyectada como una unidad productiva y de autoproducción. El marco legislativo francés, gracias a la ley ALUR, vislumbra nuevas posibilidades de construir y habitar.

Con ello, nuevas generaciones de arquitectos y ciudadanos, herederos de la reforma de la docencia de André Malraux ${ }^{53}$, parecen estar mutando hacia un perfil más simbiótico ${ }^{54}$. Ciudadanos y arquitectos parecen converger hacia la figura del grand bricoleur o hombre del bricolaje.

Este ensayo defiende una visión ecosofista ${ }^{55}$ del hombre. A través de la actividad del bricolaje y dentro de la unidad de producción de su habitar, el hombre que fabrica podría actuar a diferentes escalas: del taller a la construcción de la cité. Dicha práctica creativa del bricolaje-donde convergen técnicas corporales en la materia residual, método empírico, actividad manual e intelectual, creación y producción- parece poder intervenir en el proceso de individuación del hombre. En Francia, la aparición de la "bricoteca" podría impulsar una nueva tipología de vivienda liderada por los flujos y la transformación de materiales residuales y objetos obsoletos. Con esta premisa y contexto contingente, la vivienda del siglo XXI debe ofrecer una mayor autonomía en la gestión de flujos de materia: del circuito de agua a la basura. El espacio doméstico debe ofrecer un espacio "común" que reconcilie al hombre con el conocimiento de lo concreto: la materia en acción. Por ello, la vivienda debería imponerse como unidad productiva cuyo programa podría estar redefinido por dichos flujos. El espesor de los muros que aísla podría almacenar los materiales u objetos a la espera de un re-uso o una transformación. El taller o la "bricoteca", individual o colectivo -pero siempre en conexión con la calle-, podría ser un espacio de transición entre el interior y el exterior. Debería ser un espacio reversible, capaz de ofrecer una mayor flexibilidad ante la contingencia; por ejemplo, aislar un ciudadano enfermo en tiempos de pandemia dentro del edificio colectivo o en el seno de la casa.

Formal o informalmente, el bricolaje, junto con el espacio de taller, podría imponerse como alternativa al modelo capitalista de la obsolescencia programada. A su vez, el bricolaje como modo de construir y de habitar podría impulsar el modelo de "Ciudad de los 15 minutos"

52. GORMAN-MURRAY, Andrew. Material Geographies of Household Sustainability. Ruth Lane, Taylor \& Francis Group, 2011.

53. CHADOIN, Olivier, op. cit. supra, nota 43, p. 84.

54. ROSNAY, Joël de. L’homme symbiotique: Regards sur le troisième millénaire. París: Seuil, 1995.

55. GUATTARI, Felix. Les trois écologies. París: Galilée, 1989. 
con la que sueña el París post-COVID. Quizá, recobrando el concepto de ecosofía de Felix Guattari, no sería descabellado pensar que el hombre, gracias al bricolaje, podría redefinir su relación con el sistema Tierra ${ }^{56}$ : el bricolaje como modo de ser en el mundo. Esto podría converger con las palabras del arquitecto J. C. Quinton que defiende que la vivienda de mañana debe ser el reflejo de nuestra relación con el mundo ${ }^{57}$. En el camino hacia una transición ecológica, el bricolaje como herramienta y medio apunta a ser un catalizador de las fuerzas individuales del Homo faber hacia una re-organización económica, social y política en Francia.

\section{Bibliografía citada}

ARENDT, Hannah. The Human Condition. The University of Chicago, 1958.

AUGÉ, Marc. Non-lieux, Introduction à une anthropologie de la surmodernité. París: Seuil, 1992.

BAZIN, Hugues. L'art de Bricolage, Bricoleurs d'art. En: Les Cahiers d'Artes, L'art à l'épreuve du social. Bordeaux: Presses Universitaires de Bordeaux, 2013, n. ${ }^{9}$ 9, p. 2.

BELMONT, Alain. Les "boutiques" des artisans villageois dans la France d'Ancien Régime (XVle-début du XIXe siècle). En: Annales de Bretagne et des pays de l'Ouest, 1999, t. 106, n. ${ }^{1} 1$.

BERTHOZ, Alain. Le sens du mouvement. París: Odile Jacob, 1977.

BOURG, Dominique; PAPAUX, Alain. Dictionnaire de la pensée écologique. París: PUF, 2015.

BRAUDEL, Fernand. L'identité de la France. Les hommes et les choses. París: Arthaud, 1986.

CALVEZ, Marcel. Le bricolage dans l'aménagement de la maison. Approche à partir de 20 pavillons en lotissements HLM. París: Ministère de la Culture, 1987.

CERTEAU, Michel de. L'invention du quotidien. T. 1 y 2. [1. ed., 1980] París: Gallimard, 1990.

CHADOIN, Olivier. Le sociologue chez les architectes, matériau pour une sociologie de la sociologie en situation ancillaire. En: Sociétés contemporaines. París: Presses de Sciences Po, 2009.

DUYMEDJIAN, Raffi. La France snobe les vertus du bricolage. En: Le Monde, 2 de abril de 2013.

ELEB, Monique; SIMON, Philippe. Entre confort, désir et normes: le logement contemporain (1995-2010). Bruselas: Mardaga, 2013.

GORMAN-MURRAY, Andrew. Material Geographies of Household Sustainability. Ruth Lane, Taylor \& Francis, Group, 2011.

GOURDEN, Jean-Michel. Le peuple des ateliers. Les Artisans du xixe siècle. París: Creaphis, 1992.

GRIENER, Pascal. La notion d'atelier de l'Antiquité au xix siècle: chronique d'un appauvrissement sémantique. En: Perspective, 2014, n. 1.

GUATTARI, Felix. Les trois écologies. París: Galilée, 1989.

ILLICH, Ivan. Tools for Conviviality. New York: Harper \& Row, 1973.

JAMESON, Fredric. Le postmodernisme ou la logique culturelle du capitalisme tardif. Beaux-Arts de Paris Editions, 2007, p. 42. Texto original inglés: Postmodernism, or, the Cultural Logic of Late Capitalism. Duke University Press, 1991.

56. LATOUR, Bruno. Où atterir? Comment s'orienter en politique. París: La Découverte, 2017.

57. QUINTON, Jean-Christophe. L'urgénce à requestionner le logement. Conferencia presentada en la Cité de l'Architecture el 16 de julio de 2020. Disponible en: https://www.citedelarchitecture.fr/fr/video/lurgence-requestionner-le-logement. 
JULIEN, Marie-Pierre; ROSSELIN, Céline. La culture matérielle. París: La Découverte, 2005.

LATOUR, Bruno. Où atterir? Comment s'orienter en politique. La Découverte, 2017.

LÉVI-STRAUSS, Claude. La pensée sauvage, París: Plon, 1962.

LOGÉ, Guillaume. Art. En: BOURG, Dominique; PAPAUX, Alain, dirs. Dictionnaire de la pensée écologique. París: PUF, 2015.

MAUSS, Marcel. Les techniques du corps, sociologie et anthropologie. París: PUF, 1950 [1936]. Artículo originalmente publicado en el Journal de Psychologie, XXXII, 15 marzo-15 abril 1936, n. ${ }^{\circ}$ 3-4. Comunicación presentada a la Société de Psychologie el 17 de mayo de 1934.

NORMAND, Jean-Michel. Bricoler en temps de confinement ou la société de consolation. En: Le Monde, 27 de marzo de 2020.

PEREC, Georges. L'Infra-ordinaire. París: Seuil, 1996. Col. "Librairies du XXe siècle”.

QUINTON, Jean-Christophe. L'urgence à requestionner le logement. Conferencia presentada en la Cité de l'Architecture el 16 de julio de 2020. Disponible en: https://www.citedelarchitecture.fr/fr/video/lurgence-requestionner-le-logement.

RAGON, Michel. Histoire de l'architecture et de l'urbanisme modernes (3 t.). París: Seuil, 1991. T. 1. Idéologies et pionniers, 1800-1910. Col. “Point Essais".

REGNIER, Isabelle. Le Pritzker d'architecture pour les francais Anne Lacaton et Jean-Philippe Vassal. En: Le Monde, 16 de marzo de 2021.

RIFKIN, Jeremy. La Troisième Révolution industrielle: Comment le pouvoir latéral va transformer l'énergie, l'économie et le monde [The Third Industrial Revolution: How Lateral Power Is Transforming Energy, the Economy, and the World] (trad. del inglés). París: Les liens qui libèrent, 2012.

ROSNAY, Joël de. L'homme symbiotique: Regards sur le troisième millénaire. París: Seuil, 1995.

SENNETT, Richard. The Craftsman. Londres: Yale University Press, 2008.

SENNETT, Richard. The Open City. En: HAAS, Tigran; WESTLUND, Hans, eds. In The Post-Urban World. Londres: Routledge, 2017. Presentación en la Universidad de Harvard para el Graduate School of Design en 2013.

SENNETT, Richard. Construir y habitar. Ética para la ciudad. Barcelona: Anagrama, 2019.

SIKA, Hervé. Conférence gesticulée et dansée. Be bricoleur Baby. Extracto de la conferencia, texto dictalografiado, 2012.

WARNIER, Jean-Pierre. Construire la culture matérielle: L'homme qui pensait avec ses doigts. París: Presses Universitaires de France, 1999.

WARNIER, Jean-Pierre; BAYART, Jean-Francois. Matière à politique. Le pouvoir, les corps et les choses. Karthala, Recherches internationales, 2004.

Ophélia Mantz (Metz, Francia, 1977) es arquitecta DPLG, titulada por l'Ecole Nationale Supérieure d'Architecture de Paris-BelleviIle, ENSA-PB, Francia, y Master en Arquitectura Bioclimática y sostenibilidad por la Escuela Técnica Superior de Arquitectura de Madrid (ETSAM - UPM). Actualmente es profesora y directora del Centro de Investigación de Materiales - Material Research Center - de la escuela de arquitectura, G.D. Hines College of Architecture and Design de la Universidad de Houston, Texas, EE.UU. Co-dirige junto con Rafael Beneytez Durán, la Oficina Z4Z4 AAA (Architects Activities Associated) desarrollada como unidad productiva de diseño. Su trabajo ha sido reconocido y premiado: Architectural Review Emerging Architecture 2016 (Finalist), S.ARCH 2018, COAM 2016 Award (Finalist). La línea de investigación de su trabajo se enfoca en la construcción del pensamiento ecológico en el campo de la arquitectura y la ciudad. 
DEL BRICOLAJE AL TALLER O EL ESPACIO DE LA TRANSFORMACIÓN EN FRANCIA FROM DO-IT-YOURSELF TO THE WORKSHOP, OR THE TRANSFORMATION SPACE IN FRANCE Ophelia Mantz (https://orcid.org/0000-0003-2243-3088)

\section{p.87 INTRODUCTION}

On March 17, 2020 at noon, France decreed its first official lockdown in response to the pandemic scope of COVID-19. Prior to the ban on travel within the country, Paris, the nation's capital and the model for its historically centralized territorial policy, emptied out. The many Parisians with second homes elsewhere, along with students and young workers, already experts at surviving in the tiny dwellings available in the capital, showed no hesitation about reconquering less overpopulated rural areas. Ten days later, on March 27, Jean-Michel Normand, a journalist for the Le Monde newspaper, analyzed the practice of DIY in times of the pandemic. The article, whose title can be translated as "Do-lt-Yourself in the Lockdown Era, or the Society of Consoling,"” analyzes how the sale and use of DIY tools have been reorganizing the French people's new daily lives.

Before the COVID-19 health crisis, work and crafts performed by hand were already undergoing major growth. This had begun to occur not only among working class people, but also in the middle classes. Sociologist Ronan Chastellier links this boom in DIY (Figure 1) with the growth of a collective awareness about the reuse and recycling of objects found in our households. The abuse of planned obsolescence in everyday items seems to have led to a certain attitude in French society contrary to consumerism and, as a result, a boost in creativity.

Contemporary man is influenced by several contingency vectors that have sped up his pace of life. The climate and health crises have increased a feeling of detachment and helplessness. At the same time, the pandemic has created drastic spatial limitations with an unclear scope in time. Within this context, perhaps DIY, described by Hugues Bazin ${ }^{2}$ as an existential and contingent process, could drive us towards a new model for progress. It may be a path towards development in which man is once again equipped with handheld tools and resumes a closer relationship with

p.88 technical and productive processes. DIY may therefore constitute an opportunity to restore the people's confidence in their power to take action.

The interpretation of this moment in time may be viewed from different vantage points and territorial frameworks. However, we find it of great interest to analyze the current status of the health crisis within one specific territory and culture: France, a country that has held on to the practice of DIY as a part of its customs. Throughout this text, we will attempt to express and articulate how specific sociocultural and historical events in French culture may have triggered the current growth in DIY discussed by Chastellier. By examining this activity, considered by many to be a space of resistance, we get a glimpse of a movement towards seeking new ways to inhabit domestic space, to "be in the world" or être-au monde.

In this essay, we will first analyze the characteristics of and changes across time in the activities related with doit-yourself work. In doing so, the emphasis will be placed on the potential action this activity may exert on the process of man's individuation. Secondly, we will envisage DIY's potential role in shifting the current economic and productive model towards a more sustainable system, in what is known as the ecological transition. In the third section, we study the historical role of the workshop space as a construct in France's social and urban fabric. Last of all, parallels will be drawn between the renewed appreciation for DIY and newly defined profiles of contemporary French architects, who have inherited profound curricular changes that gained traction in the seventies.

The article's hypothesis lies in demonstrating that emerging practices in the realm of French architecture may also be resulting from a world which is re-assembling items that already exist while giving value to the obsolete. In our contemporary era, DIY seems to be taking over as a tool and means for change towards building a new paradigm. In doing so, we shall highlight the need to breathe life into workshops and seek a comeback in workshops as spaces for shared co-existence as a new dimension in twenty-first century housing.

\section{DO-IT-YOURSELF: ORIGIN AND EVOLUTION OF A MARGINAL ACTIVITY}

The French word bricolage ("do-it-yourself work") is derived from the Italian word briccola, used in the Middle Ages around 1360 with the meaning of "catapult." The word's etymological origin can also be found in the root of the French word bricoles, which means a set of leftover items or accessories with little value. Beyond this some sometimes pejorative connotation, bricolage often tends to refer to the activity of creating self-made items, reflecting a privileged relationship between inhabitants and objects ${ }^{3}$.

In the twentieth century, French ethnology, sociology and anthropology ${ }^{4}$ were influenced by studies on everyday activities. DIY is linked to domestic space as an expression of multi-purpose techniques employed to use leftover materials and items. Many researchers have valued the process of individuation entailed by this sometimes marginalized activity. Herein the term individuation refers to the psychological construction of an individual seeking to articulate bodily subject and society. Since the 1990s, thanks to the culture of do-it yourself in the English-speaking world, DIY has been identified as both a potential business activity and an activity of resilience ${ }^{5}$. Within the French cultural framework, this admittedly marginal activity has been a focus of interest for several decades. From Michel de p.89 Certeau ${ }^{6}$ to Georges Perec ${ }^{7}$, DIY symbolizes a space of resistance against the production logic of consumerist society. 
Perhaps lying within this realm of contingency is the path towards a model of society described by Ivan Illich as that of conviviality ${ }^{8}$. Illich refers to a social structure in which the rights to use the energy to work and live in a creative way are ensured. DIY inspires and raises questions about man's relationship with everyday objects. Its premises lie in indeterminacy, uncertainty, contingency, chance and chaos, all of which enshrine it as a symbol of the process itself, with no specific project. DIY is perceived as the means to achieve creative autonomy; it rejects any separation between research and action, manual and intellectual activity, creation and production. In other words, based on a strictly empirical approach, man as assembler, freed from subordination, ceases projecting a hegemonic vision upon reality, and instead invents a new way of looking at it.

In his book La pensée sauvage (The Savage Mind), structuralist anthropologist Claude Lévi-Strauss establishes an analogical relationship between primitive science and DIY. He defines it as a technique of the concrete. In the first chapter, titled "The Science of the Concrete," he recalls how, in the Neolithic period, man's practice gradually formed in the great arts of civilization such as pottery, the loom, agriculture and the domestication of animals. All of these techniques were the result of centuries of active, methodical observation ${ }^{9}$ after repetitive experiences and perseverance. DIY is described as a manual activity, the result of which builds upon knowledge of the concrete. Practicing DIY consists of assembling irregular, leftover items after the bricoleur, or do-it-yourselfer, has sorted them out. This activity reveals the ability to repurpose the objects being re-assembled and manipulated from their original use. In other words, with no previous biases, the do-it-yourselfer seeks to create a new order and give cohesiveness to a set of objects, tools and information that he has acquired for himself; he offers a solution based on a versatile body of knowledge about the places to be intervened in, knowledge about the objects and the materials of which they are composed. One of the features of DIY is recovering unused materials grouped together before the project begins, forming a new source of available raw materials. Behind this surely self-taught activity, man takes pleasure in managing the economy of means, increasing his ability to invent and act as the sole role-player in the building process.

In another comment, Lévi-Strauss discusses the mythical-poetic dimension of DIY,10 establishing analogies with Postman Cheval's castle and with the special effects created by Georges Méliès (Figure 2). All of the items which comprise the inventory of the bricoleur, do-it-yourself man, become a treasure that may give birth to an aesthetic world of great value. DIY is made up of the leftovers from human works, regarded as a sous-ensemble or subculture ${ }^{11}$. Anthropologists define DIY as the creation of structures by assembling items left over from events, bits and pieces, bearing witness to the history of an individual or society ${ }^{12}$. Lévi-Strauss established a relationship between mythical or magical thinking and DIY. He reminds us that an artist is born when the wise man meets the DIY world ${ }^{13}$.

DIY is of interest to us to the extent that it reveals or reminds man of his ability to find solutions to concrete problems. This value is personified by the craftsman, as described by Richard Sennett ${ }^{14}$ in his book The Craftsman. p.90 The re-use and assembly of objects from the economy of leftover materials provide a qualitative value through wideranging technical knowledge. We are concerned with seeing how man acquires bodily techniques when performing this non-professional activity which reveal his ability to act upon objects. His physical body becomes engaged with material cultural, defined by ethnologist J. P. Warnier and sociologist J. F. Bayart as a vehicle for subjectivization insofar as it contributes to the formation of moral subjects and the production of social relations ${ }^{15}$. In other words, man's physical relationship with matter forms part of the process of subjective and collective individuation ${ }^{16}$.

According to Hervé Sika ${ }^{17}$, the activity of DIY consists of giving art a relational dimension. He also reminds us that the reality of the do-it-yourselfer is heterogeneous, de-hierarchicalized and liberating. Thus, DIY places the subject in a relationship with leftover objects. The individual transforms them through bodily techniques ${ }^{18}$ acquired by experience or learned in other settings. These techniques of the body make it possible to sort and find a new appreciation for the original materials. The body thinks ${ }^{19}$ and can signify the value of these materials through its gesture ${ }^{20}$ within a system of connotations and communication. In other words, the body is a material in and of itself, which works and is worked ${ }^{21}$; it produces and is a product.

\section{DO-ITTOURSELF: A TOOL FOR TRANSFORMATION TOWARDS AN ECOLOGICAL TRANSITION}

Within the context of the current pandemic, "action" taken on matter, represented at the domestic scale of DIY, appears to be an opportunity for change as we move towards an ecological transition. In this sense, DIY heightens the need to learn how to deal with contingencies and offers us the chance to become role-players in a manual production process $^{22}$. In accordance with terms laid down by Richard Sennett, the activity of DIY could be considered the emergence of new ways of moving towards collective action ${ }^{23}$ : action taking place before awareness.

This activity may perhaps also be an opportunity to put an end to the hegemony of "the eye," or the visual as the supreme sense ${ }^{24}$ that alienates us. In order to more deeply examine the idea that "action upon matter" is a decisive stage in man's evolution, we will use the argument by ethnologist Jean-Pierre Warnier based on "thinking with our 
fingers." This stance is also defended by architects such as Juhani Pallasmaa in his book The Thinking Hand in 2009 or Flores and Prats in Pensado a mano.

In his book, Construire la culture matérielle: L'homme qui pensait avec ses doigts ${ }^{25}$, J. P. Warnier explains how

p.91 bodily techniques and dynamic engagement in material culture are articulated with representations of the world that give man his identity. The author describes the process of hominization through the relationship established with matter. He uses the idea of cultural-bipedal hominization put forth by primatologist Toshisada Nishida. As a result of his studies on chimpanzees, this Japanese pioneer emphasized the importance of cultural behavior in animals, leading to the opening of a new "ecological niche." The creation of an ecological niche means choosing a specific environment, as well as knowing what elements allow life and reproduction of the species in accordance with new systems. In other words, the creation of an ecological niche takes place along with the acceptance of new rules in the game of existence $^{26}$. Warnier argues that tools, as part of man's evolution, are the result of a motor-based decision reached in the face-off between society, culture and environment ${ }^{27}$, and therefore, according to Nishida, they did play a role in defining a new ecological niche. For these reasons, we propose viewing in DIY activity the potential for creating a niche that drives new rules, values and behaviors while moving towards a more sustainable development model. This would be the result of constructing new means of existence for man that match up with DIY as a tool for action and a means for being in the world.

In order to contribute a reflection about the limitations of contemporary domestic space types in France, and perhaps in other countries, this article proposes considering the possibility of "reintroducing" the DIY space, a place for assembling leftover materials, into household designs. To examine this topic with historical depth, the role of the workshop space in France prior to the industrial revolution is then studied and analyzed. This analysis allows us to explore the possibility of reintegrating a space that begins inside the dwelling and then becomes a feature helping to build the urban fabric: from the workshop to la cité.

\section{HOMO FABER, OR HOW TO BUILD LA CITÉ FROM THE WORKSHOP}

Before being exclusively attributed to the artist, the workshop or atelier shifted between different terms such as boutique or échoppe (Figure 3), all meaning: the craftsman's work space. In any case, these terms have always been laden with commercial connotations. In one case or another, these spaces have always represented the spatial dimension for the production of objects. It is there where man, craftsman or artist has assembled and transformed materials and/

p.92 or objects. This fate of the materials in these objects is basically supported by the implementation of techniques using the body. As mentioned above, within the space of the workshop, the human body is put into action upon matter and is capable of giving new value to leftover materials. Marcel Mauss, the father of French ethnology, defined these bodily techniques as the ways in which men passed on knowledge about how to use their bodies from one society to the next in a traditional manner. The workshop space, whether directly or indirectly connected to the domestic space, made it possible to heighten the creative qualities of a species of man known as homo faber ${ }^{28}$. Urbanist Richard Sennett reminds us that men who produce things are respected in the city through modest practice. Homo faber adheres to a production ethic that implies a specific relationship with la cité. The primacy of la cité came about with the production that arose from habitation ${ }^{29}$. Homo faber instigates a behavior, an ethic from which rises forth the idea of la cité. For Sennett, producing constitutes la cité, whereas la ville is habitation. But in the twentieth century, cité and ville grew apart. As a result, homo faber, the social role-player in the city, fell to pieces.

Up to the industrial revolution, men who made tools were the main role-players in social organization. However, despite the advent of the machine-and with it the sector-based division of work in the building process-the development of the nineteenth-century industrial economic model did not manage to do away with workshop in one fell swoop. The process of its disappearance was much slower and more gradual. These features of the resilient character of the bricoleur reinforce the idea that the space where materials are transformed may be a space of resistance against the production logic. The workshop, a symbol of the craftsman's world, persevered and remained a structure-building space in terms of the types of units in habitations, extending into territorial occupation. This space took over as a vector of organization on a larger scale in the rural habitat, as well as in the urban fabric ${ }^{30}$ (Figures 4 and 5). The workshop, whether separate or connected to the domestic space, played a role in French social and economic organization until the end of the nineteenth century.

It is worth being reminded that, in the transition from prehistory to history, the technical handling of materials like stone, bronze and clay, generated a body of craftsmanship from which different societal models were established ${ }^{31}$. Because of this, the workshop is understood to be the spatial representation of the craftsman's world. In the nineteenth century, the Dictionnaire raisonné de l'architecture française du siècle XI au XVI, by architect Eugène Viollet-le-Duc, provided several descriptions of the boutique or shop, as types of domestic spaces. In cities as important as Paris and in the less populated urban areas that make up the rural world, the workshop, or boutique, forms an integral part of the architecture in the landscape ${ }^{32}$ (Figures 6 and 7). The workshop, so closely linked to domestic space, became more individualized as a work space as history moved forward. Because of its location on the façade, the workshop-

p.93 space was expressed architecturally and, therefore, became legitimized in the public space of the street. In doing so, it became a basic feature in the construction of urban centers in France. In other words, this transformation, which made the craftsman's professionalization apparent, enhanced the presence of the workshop-boutique as a role-player within the urban environment ${ }^{33}$. 
J.-M. Gourden in his book Le peuple des ateliers. Les artisans du xix siècle (The People of the Workshops: Craftsmen of the nineteenth century) describes how workshops represented France's economic and social organization before and after the industrial revolution. The workshop remained a feature of the urban factory nearly until the turn of the twentieth century. However, the advent of technical progress due to the steam engine-and with it the proletariatdestabilized the workshop's role in the system. Craftsmen experienced the industrialization process as a form of dispossession ${ }^{34}$. Their lengthy agony gradually led to the disappearance of workshops as they shut down in favor of factories for nearly an entire century. However, the slow nature of their demise also demonstrates that, beyond just housing manual activity, workshops had been a space of resistance.

According to philosopher Hannah Arendt, the takeover of the industrial model turned the craftsman, also described as homo faber, into an animal laborans ${ }^{35}$. This definition seems to indicate a negative, dehumanizing transformation of man in the nineteenth century. Man, historically the protagonist of tool making, was turned into a slave within the production process forced upon him by industrialization. The workshop or productive unit, where man carries out his process of subjective, social individuation by using bodily techniques and tools on materials, vanished throughout the nineteenth century. The term boutique or shop moved into the realm of the mechanical arts. As a result, it is the chantier, or work site, which came to define the workman's space. The workshop space survived solely and exclusively to meet the needs the artist's existence ${ }^{36}$. This mutation in design succumbed with the publication of the Encyclopédieor Dictionnaire raisonné des sciences, des arts et des métiers, by Denis Diderot (1713-1784) and Jean Le Rond d'Alembert (1717-1783)-, which forever legitimized its purpose as exclusively artistic. The dictionary that symbolizes the appetite for knowledge in eighteenth-century France, the century of enlightenment or siècle des lumières, ushered in a profound redefinition of society and its material forms ${ }^{37}$.

As we have seen above, the nineteenth and twentieth centuries eliminated the workshop as a space within France's urban factories and saw the disappearance of homo faber as a role-player in la cités ${ }^{38}$. However, DIY activity continued to be performed within domestic spaces. DIY, regarded as a poorly defined, marginal activity, became a subject of study in the human and social sciences during the second half of the twentieth century.

The future of a space for transformation or DIY activity converged with Guillaume Logé's praise-in the Dictionary of Ecological Thought ${ }^{39}$-of creative integration as an economic model able to meet man's need for meaning ${ }^{40}$. The author mentions economist Jeremy Rifkin ${ }^{41}$, who in 2011 advocated that a third industrial revolution might occur. With this theoretical position, the American economist/ecologist established sustainable economic models based on innovation and value creation. These models would give rise to what he calls cultural capitalism. This new, more horizontal economic model is driven by the Internet, the emergence of new domestic tools and a reduction of the value chain. This new framework for action would lead to the development of a distributed, cooperative economy ${ }^{42}$. It is a model that is to give man greater autonomy, instead of keeping him under control.

\section{THE GRAND BRICOLEUR: A PARADIGM SHIFT FOR FRENCH SOCIETY}

Over a period of fifty years, the development and advancement of sociology, anthropology, ethnology and philosophy have impacted the curricular structure at architecture schools in France. In the years of 1967-68, France underwent a student revolution known as May 68, which led to major changes in post-war French society. It was within this context that France's Minister of Cultural Affairs, André Malraux, decided to reform education in France. Max Querrien, with the help of Henri Raymond, a close friend of Henri Lefebvre's, decided to introduce the human and social sciences into architecture studies ${ }^{43}$. He cultivated a profile of the "sociologist architect" who could ease this transition in household space.

Despite the impact of social sciences on the curriculum at French architecture schools, the types of domestic space produced have only timidly included new lifestyles in the twenty-first century. According to Monique Eleb-regarding the types of housing supplied ${ }^{44}$-despite the fact that the French architect's power to take action has been shifted towards developers, new generations of professionals seem ready and willing to offer new ways of thinking about housing in the twenty-first century. It is worth noting that the sensitivity of architects trained from a multidisciplinary viewpoint of inhabited space allows us to envisage new spatial models, as well as professional practices that enshrine the concept of "making more space with fewer means." Perhaps the context of the pandemic might stimulate and legitimize the emerging practices of a new generation of French architects.

At the end of the twentieth century, architects Anne Lacaton and Jean-Philippe Vassal promoted a vector of change through various housing projects: doing more with less, and making things with others that already exist by reducing demolitions. The Latapie House, built in Floirac, France in 1993, surely displays the most emblematic of strategies in the building of new domestic spaces (Figures 8 and 9). Undoubtedly, its ingenuity in the use of materials borrowed from the rural farming world, coupled with its creation of a non-hierarchicalized, heterogeneous space, have made it possible to transgress many semantic codes in household space. It should also be noted that the house has also made it possible to display an independent, unprejudiced spirit. Isabelle Regnier, a journalist for Le Monde, describes the works by this tandem of architects as being filled with a certain "do-it-yourself charm ${ }^{45}$."

At the same time as these experiments on "making more with less" performed by Lacaton and Vassal, in Marseille, France, a project called La Friche la Belle de Mai46 was started up in 1992. This project in constant transformation illustrates change in the manner of inhabiting and the construction of the urban factory. The project has been defined as a political experiment, and a place for thought and action about redefining the relationship between art, territory 
and society. The project, heterogeneous and certainly informal, arose from the recovery of an old factory. Its genesis, grounded in the artistic activity of different role-players (artists, residents, intellectuals), follows certain guidelines from

p.97 the activity of DIY: anticipating without making unchangeable decisions, building with flexibility, experiencing and encouraging a spirit of cooperation. Several French architects have taken part, including Matthieu Poitevin and Pascal Reynaud, and the studio Agence Construire-Sébastien Eymard et Loïc Julienne, with Simon Himpens.

Along the path towards the grand bricoleur, another event closer to the present day occurred in the French pavilion at the 2018 International Architecture Exhibition in Venice (an exhibition curated by Irish architects Yvonne Farrell and Shelley McNamara). In principle, the pavilion installation has had a limited media impact, but in this article we are interested in culturally re-contextualizing what might have been emerging: while the Swiss pavilion praised the void, the French pavilion (Figures 10 and 11), designed by the Encore Heureux studio, interprets the theme of freespace ${ }^{4747}$ from a humanistic perspective. The Parisian architectural firm's members defended the role of the architect as a driver of the ecological transition.

The pavilion exhibited a huge panoply of everyday objects. By preserving, recovering and reusing objects, materials, structures, and so forth, the architect becomes the role-player who arranges and re-organizes everything that already exists. The role of an architect who navigates amid everyday objects and assembles them is thereby promoted. He becomes the man of a larger-scale DIY, capable of giving value to available materials above and beyond the semantic burden held by manufactured materials. In other words, by looking at this example, what is of interest to us is recovering the original idea presented at the beginning of this article, in which bodily action on matter produces qualitative value in man's material world.

In accordance with the paradigm shift in French society, the legitimacy of participatory housing could become a significant development as part of the concept of self-production. In 2014, a new concept of housing, defined as participatory and known as logements participatifs ${ }^{48}$, was legislated through a law called the ALUR Act. This new model, though still being developed, follows in the footsteps of countries such as Germany, Switzerland or Norway.

p.98 In France, the ALUR Act has modified the home building code. This regulation combines legislative and regulatory frameworks related with construction, property development and social housing in France.

The ALUR Act highlights the desire to offer new ways of living in groups during an era of ecological transition prior to the COVID-19 health crisis. Thanks to the increase in political momentum, the social union for habitation, or Fabrique de la ville et Transit City, presented a report in November 2020, in which it proposes the institutionalization of DIY ${ }^{49}$. The pandemic seems to have sped up projects already under way. It may even have placed a greater emphasis on them, and because of the health crisis, politicians have finally legitimized them. The spaces known as fab labs (Figure 12), living labs and other makerspaces have taken over in French territory, officially receiving a financial outlay of 110 million euros from the State on September 19, 2020.

Due to the impetus which social sciences in the post-war years gave to the legitimization of DIY spaces in the midst of the pandemic, through the emergence of bricoleur-architects, everything seems to indicate a significant shift in French society. It all makes clear the need to acknowledge other ways of making things and living. In the specific case at hand, the desire to institutionalize the DIY space, in the form of a DIY library or bricothèque ${ }^{50}$ (Figures 13 and 14), is undoubtedly a constituent stage within a paradigm shift towards ecological transition.

Bringing back the workshop/studio could lead to greater flexibility in the design of living spaces. This neutrality is more consistent with the contingencies of life. In short, one could claim that domestic space is no longer viewed so much as a dwelling, but rather as a productive unit.

Political support in France, probably driven by the health and environment crises, has allowed for a new appreciation of these creatively integrated spaces, which have gone from being an alternative, marginal phenomenon to an avant-garde space of resistance and self-production.

\section{CONCLUSIONS}

The new characteristics of French living spaces could be described as hybrid, reversible and diverse. Thanks to miniaturized new tools created by big industry-3D printers, laser cutters, digital equipment, etc.-homo faber seems to have risen from his ashes. Innovative, autonomous, resilient man has come into being as a result of the sacrifice by animal laborans or the proletariat of the nineteenth and twentieth centuries. Animal laborans has given up his individuality in order to form part of a social body executed in the world of the factory. However, the concept of resistance which characterizes homo faber ${ }^{51}$ has turned him into the main role-player within a deeper political transformation towards ecological transition. The new space of the "DIY library" or bricothèque seems to have the ability to float amid different

p.99 scales of existence: from the domestic realm to institutionalized public space. In both cases, DIY, as a multi-purpose technical activity originating in the working classes, seems to indicate a profound change in French society: the building of a new ecological niche.

The common space of the workshop creates the potential for bringing back a space for transforming matter in the new types of housing built in the twenty-first century. However, what we are discussing is a potential, informal, heterogeneous space that can come in many forms, privileging the act of transforming matter and, by doing so, its semantic value, as well. Such a space seems to make sense as a part of contemporary domestic life, with all its accumulation of objects and leftover material flows ${ }^{52}$. Beyond the workshop, though, homes could be designed as 
productive units for self-production. Because of the ALUR Act, the French legal framework has made room for new possibilities in building and inhabiting.

In doing so, new generations of architects and people, the products of André Malraux's teaching reform ${ }^{53}$, seem to be mutating to engage in greater symbiosis ${ }^{54}$. Both people and architects seem to be merging into the figure of the grand bricoleur, or do-it-yourself man.

This essay defends an ecsophist ${ }^{55}$ view of man. Through the activity of do-it-yourself within the production unit formed by his dwelling, the man who produces may be able to act at different scales: from the workshop to construction of la cité. This creative practice of do-it-yourself-in which bodily techniques converge in leftover materials, the empirical method, manual and intellectual activities, creation and production-appears to have the ability to play a role in man's individuation process. In France, the emergence of the bricothèque may give rise to new types of housing designed in accordance with the flows and transformation of leftover materials and obsolete objects. With this premise and contingent context in mind, twenty-first century housing must provide greater autonomy in terms of managing material flows: from the water cycle to waste. Households must provide a "common" space that allows man to come to terms with knowledge of the concrete: putting matter in action. Therefore, housing should be turned into a productive unit whose design can be redefined by these flows. Thick space in insulating walls could be used to store materials or objects awaiting reuse or transformation. The workshop or "DIY library," whether individual or collective-though always in connection with the streets outside-could become a transitional space between interior and exterior. It should be a reversible space that provides greater flexibility to deal with contingencies; for example, when isolating someone ill inside of a collective building or house during the pandemic.

Whether formally or informally, DIY, combined with the workshop space, could take over as an alternative to the capitalist model of planned obsolescence. In turn, DIY as a way of building and inhabiting could drive the model of the "15-minute city" that post-COVID Paris has been dreaming of. Perhaps, recovering Felix Guattari's concept of p.100 ecosophy, it might not be outlandish to think that, because of DIY, man may be able to redefine his relationship with the Earth system ${ }^{56}$, making do-it-yourself a way of being in the world. This could mesh with the words of architect J. C. Quinton, who argues that tomorrow's housing should be a reflection of our relationship with the world ${ }^{57}$. DIY, as a tool and means used on the path towards ecological transition, appears to be acting a catalyst for the individual strengths of homo faber in moving towards an economic, social and political reorganization in France.

1. Normand, Jean-Michel. Bricoler en temps de confinement ou la société de consolation. In: Le MondeMarch 27, 2020.

2. BAZIN, Hugues. L'art de Bricolage. Bricoleurs d'art. In: Les Cahiers d'Artes. L'art à l'épreuve du social. Bordeaux: Presses Universitaires de Bordeaux, $2013, n^{\circ} 9$, p. 2.

3. Calvez, Marcel. Le bricolage dans l'aménagement de la maison. Approche à partir de 20 pavillons en lotissements HLM. Paris: Ministère de la Culture, 1987. This article presents the results of a research project financed by the "Mission du Patrimoine Ethnologique."

4. Warnier, Jean-Pierre. Construire la culture matérielle: L'homme qui pensait avec ses doigts. Paris: Presses Universitaires de France, 1999. The author, an ethnologist, provides a wide-ranging bibliography, from Marcel Mauss to Michel de Certeau, Bourdieu, Baudrillard and Latour.

5. Duymedjian, Raffi. La France snobe les vertus du bricolage. In: Le MondeApril 2, 2013. This articles refers to Karl Weick, a professor of psychology and management at the University of Michigan's Ross School of Business, USA.

6. Certeau, Michel de. L'invention du quotidien. T. 1 and 2. [first edition,1980] Paris: Gallimard, 1990.

7. Perec, Georges. L'Infra-ordinaire. Paris: Seuil, 1996. From the collection "Librairies du xx siècle".

8. Illich, Ivan. Tools for Conviviality. New York: Harper \& Row, 1973.

9. Lévi-Strauss, Claude. La pensée sauvage. Paris: Plon, 1962, p. 20.

10. Ibid., p. 29.

11. Ibid., p. 31.

12. Ibid., p. 34

13. Ibid., p. 35.

14. Sennett, Richard. The Craftsman. London: Yale University Press, 2008.

15. Warnier, Jean-Pierre; Bayart, Jean-François. Matière à politique. Le pouvoir, les corps et les choses. Paris: Karthala, 2004, p. 319. From the collection "Recherches internationals."

16. Julien, Marie-Pierre; Rosselin, Céline. La culture matérielle. Paris: La Découverte, 2005, p. 65.

17. Sika, Hervé. Conférence gesticulée et dansée. Be bricoleur Baby. Excerpt from the conference, typed text, 2012.

18. Mauss, Marcel. Les techniques du corps, sociologie et anthropologie. Paris: PUF, 1950 [1936]. Article originally published in the Journal de Psychologie, XXXII, March 15-April 15, 1936, n 3-4. Paper presented to the Société de Psychologie on May 17, 1934.

19. Warnier, Jean-Pierre, op. cit. supra, Note 4.

20. Berthoz, Alain. Le sens du mouvement. Paris: Odile Jacob, 1977. The author studied how the gesture made by workers from the charitable association EMMAÜS, at the behest of Abbé Pierre (a French bishop who defends the poor) put leftover clothes and objects to new uses little by little. This highlights the importance of movement in general and the gestures made by workers in the sorting process, making it possible to lend new uses to unused materials.

21. Julien, Marie-Pierre; Rosselin, Céline, op. cit. supra, Note 16.

22. Arendt, Hannah. The Human Condition University of Chicago Press, 1958, p. 285.

23. Sennett, Richard. The Open City. In: Haas, Tigran; Westlund, Hans, editors, In The Post-Urban World. London: Routledge, 2017. Presentation at Harvard University for the Graduate School of Design in 2013.

24. Jameson, Fredric. Le postmodernisme ou la logique culturelle du capitalisme tardif. Paris: Beaux-Arts de Paris Editions, 2007, p. 42. Original English text: Postmodernism, or, the Cultural Logic of Late Capitalism. Duke University Press, 1991.

25. Warnier, Jean-Pierre, op. cit. supra, Note 4. 
26. Ibid., p. 44

27. Ibid., p. 47.

28. Bourg, Dominique; Papaux, Alain. Dictionnaire de la pensée écologique. Paris: PUF, 2015, pp. 536-540.

29. Sennett, Richard. Construir y habitar. Ética para la ciudad. Barcelona: Anagrama, 2019, pp. 14-18.

30. Belmont, Alain. Les "boutiques" des artisans villageois dans la France d'Ancien Régime (xve'-début du xixe siècle). In: Annales de Bretaǵne et des pays de l'Ouest, 1999, Tome 106, $n^{\circ} 1$, pp. 197-210.

31. Braudel, Fernand. Lidentité de la France. Les hommes et les choses. Paris: Arthaud, 1986.

32. Belmont, Alain, op. cit. supra, Note 30.

33. Idem.

34. Gourden, Jean-Michel. Le peuple des ateliers. Les Artisans du xxe siècle. Paris: Creaphis, 1992.

35. Arendt, Hannah, op. cit. supra, Note 22, pp. 327-337.

36. Griener, Pascal. La notion d'atelier de l'Antiquité au xxe siècle: chronique d'un appauvrissement sémantique. In: Perspective, 2014, nº 1, p. 20. The author reminds us that Diderot and D'Alembert's Encyclopédie gradually redefined the atelier (workshop) as a space exclusively attributed to artists, reserving for workmen a relationship with the chantier or work site; the space known as the boutique or shop was attributed to the mechanical arts.

37. Ragon, Michel. Histoire de l'architecture et de l'urbanisme modernes (3 tomes). Paris: Seuil, 1991. T. 1. Idéologies et pionniers, 1800-1910. From the collection "Point Essais."

38. Sennett, Richard. Construir y habitar. Ética para la ciudad. Barcelona: Anagrama, 2019, pp. 14-18.

39. Bourg, Dominique; Papaux, Alain. Dictionnaire de la pensée écologique. Paris: PUF, 2015, pp. 61-64.

40. Augé, Marc. Non-lieux. Introduction à une anthropologie de la surmodernité. Paris: Seuil, 1992.

41. Rifkin, Jeremy. La Troisième Révolution industrielle: Comment le pouvoir latéral va transformer l'énergie, l'économie et le monde [The Third Industrial Revolution: How Lateral Power Is Transforming Energy, the Economy, and the World] (French translation from the English). Paris: Les liens qui libèrent, 2012, 380 p. The book was highly criticized in France by philosopher Dominique Bourg and environmental politician Noël Mamère.

42. Logé, Guillaume. Art. In: Dictionnaire de la pensée écologique. Bourg, Dominique; Papaux, Alain, directors. Paris: PuF, 2015, pp. 61-64.

43. Chadoin, Olivier. Le sociologue chez les architectes, matériau pour une sociologie de la sociologie en situation ancillaire. In: Sociétés contemporaines Paris: Presses de Sciences Po, 2009, p. 84. In 1968, reforms at the traditional Fine Arts School made it possible to create independent Ateliers d'Architecture which gave rise to today's ENSAs (National Higher Schools of Architecture).

44. Eleb, Monique. Entre confort, désir et normes: le logement contemporain (1995-2010) Brussels: Mardaga, 2013.

45. Regnier, Isabelle. Le Pritzker d'architecture pour les francais Anne Lacaton et Jean-Philippe Vassal. In: Le Monde' March 16, 2021.

46. Available at: https://www.lafriche.org/.

47. Venice Architecture Biennial, from May 26 to November 25, 2018. Available at: www.labiennale.org/en.

48. Participatory housing is based on a citizen-centered focus: it allows groups of people to build their homes and share an ecological, community-based lifestyle at a lower cost. It is included within the ALUR Act. The goal of the cooperative framework is to change the way of living in and organizing buildings, making their inhabitants active, and thus fighting against speculation.

In this law, participatory housing is defined with a citizen-based focus that makes it possible for private individuals and bodies corporate to team up in order to take part in the definition and design of their homes and other facilities. All of them play a role in the decisions reached on the construction and acquisition of the building and, where appropriate, building management. Available at: https://www.ecologie.gouv.fr/habitat-participatif-cadre-juridique-habiter-autrement.

49. Velez, Véronique; Demery, Cécile; Mercurio, Céline Di. Etude prospective: Nouveaux usages et modes d'habiter [online]. Paris: L'Union Sociale pour l'Habitat, 2020 [consulted on: Oct. 7, 2021]. Available at: https://www.union-habitat.org/centre-de-ressources/patrimoine-maitrise-d-ouvrage/nouveaux-usages-et-modes-d-habiter-analyse-d.

50. Ibid., p. 24

51. Arendt, Hannah, op. cit. supra, ${ }^{\text {Note } 22,0.0332}$

52. Gorman-Murray, Andrew. Material Geographies of Household Sustainability Ruth Lane, Taylor \& Francis Group, 2011.

53. Chadoin, Olivier, op. cit. supra, Note 43, p. 84.

54. Rosnay, Joël de. L'homme symbiotique: Regards sur le troisième millénaire. Paris: Seuil, 1995.

55. 55 Guattari, Felix. Les trois écologies: Paris: Galilée, 1989.

56. Latour, Bruno. Où atterir? Comment s'orienter en politique. Paris: La Découverte, 2017.

57. Quinton, Jean-Christophe. L'urgence à requestionner le logement. Conference given at the Cité de l'Architecture on July 16, 2020. Available at: https://www.citedelarchitecture.fr/fr/video/lurgence-requestionner-le-logement. 


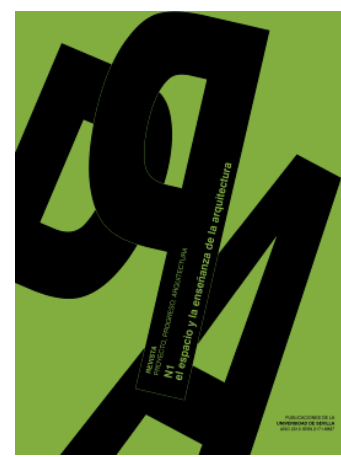

PpA N01

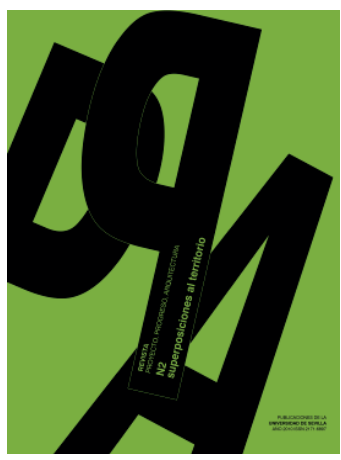

PpA N02

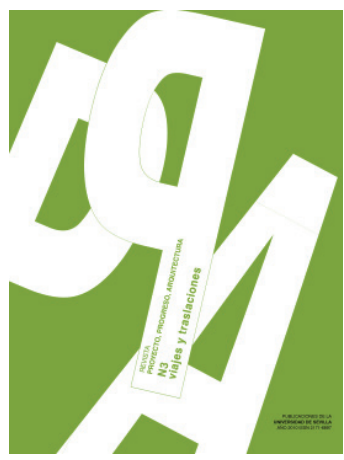

PpA N03

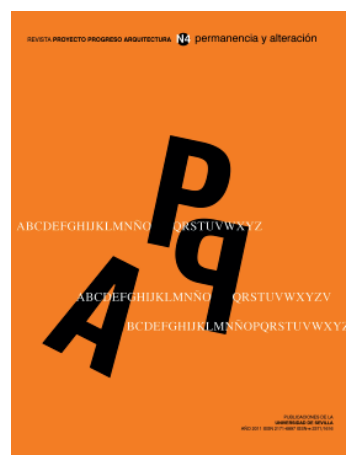

PpA N04

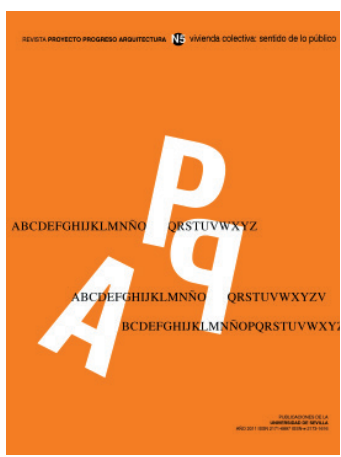

PPA N05

N01. EL ESPACIO Y LA ENSEÑANZA DE LA ARQUITECTURA (mayo, 2010) / N02. SUPERPOSICIONES AL TERRITORIO (mayo 2010) / N03. VIAJES Y TRASLACIONES (noviembre 2010) / N04. PERMANENCIA Y ALTERACIÓN (mayo 2011) / NO5. VIVIENDA COLECTIVA: SENTIDO DE LO PÚBLICO (noviembre 2011)

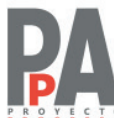

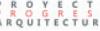

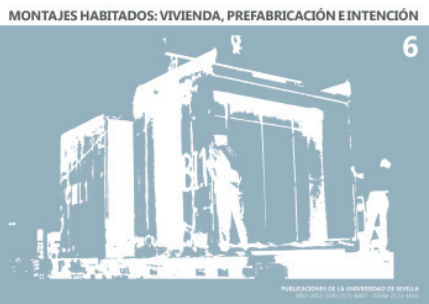

PpA N06

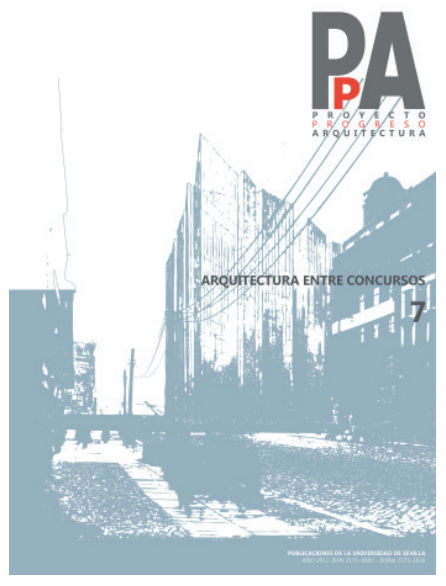

PpA N07

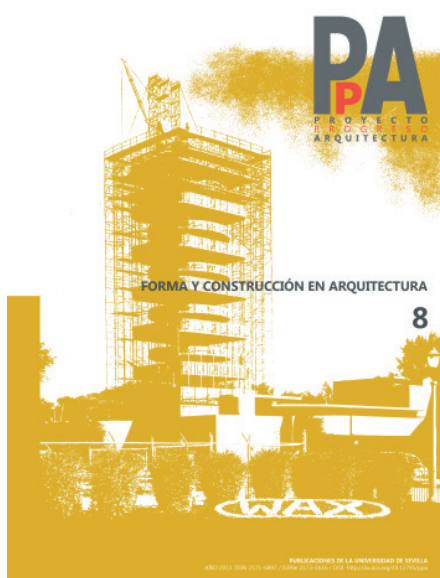

PpA N08

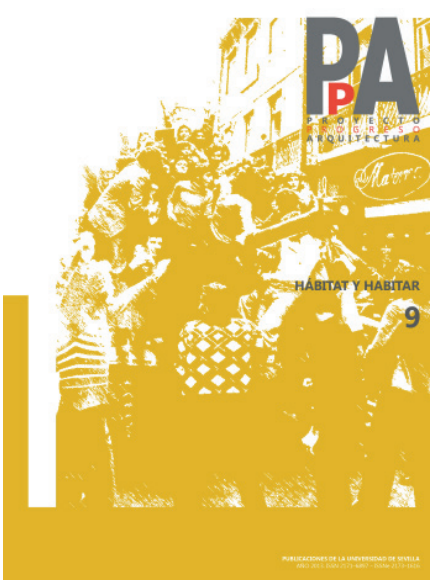

PpA N09

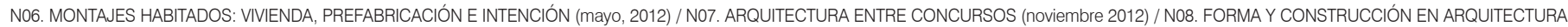
(mayo 2013) / N09. HÁBITAT Y HABITAR (noviembre 2013)

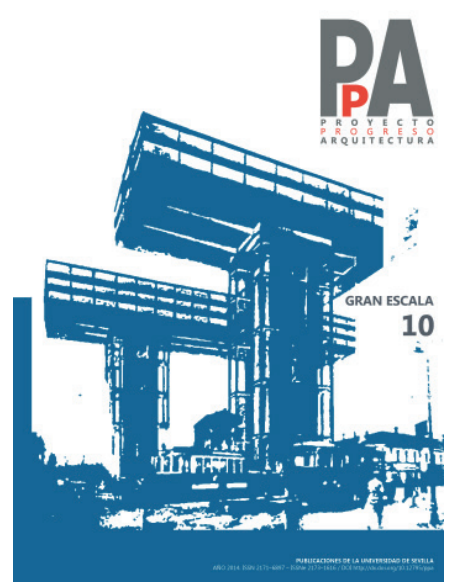

PpA N10

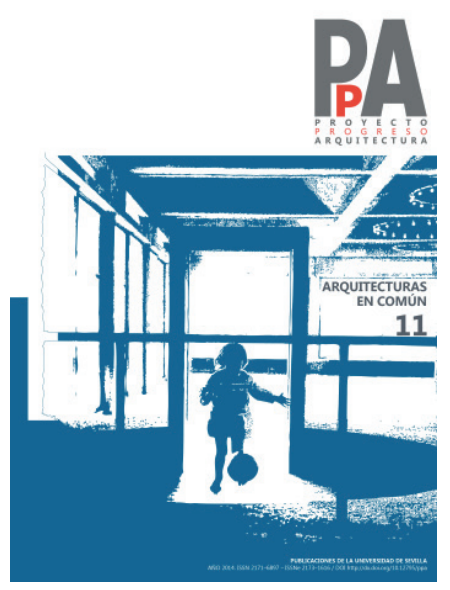

PpA N11

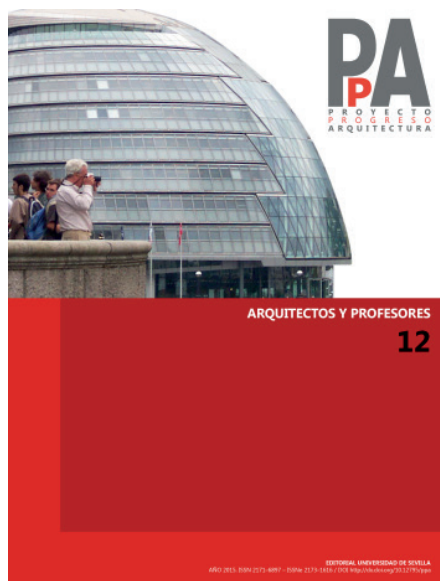

PpA N12

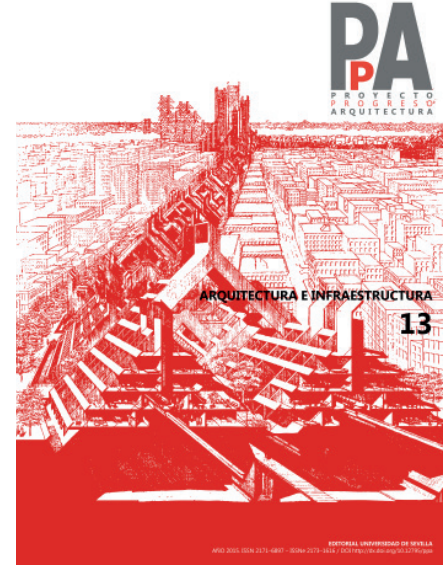

PpA N13

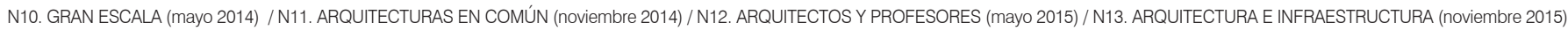




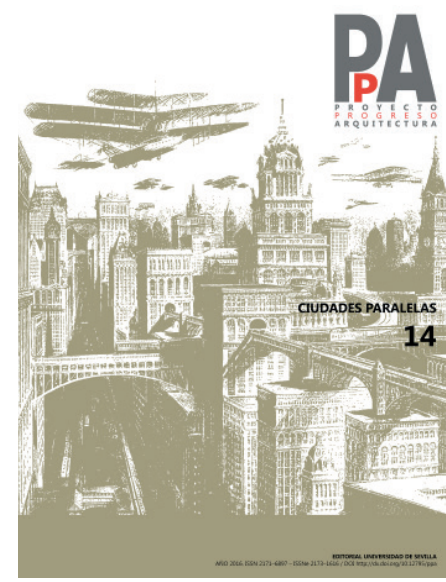

PpA N14

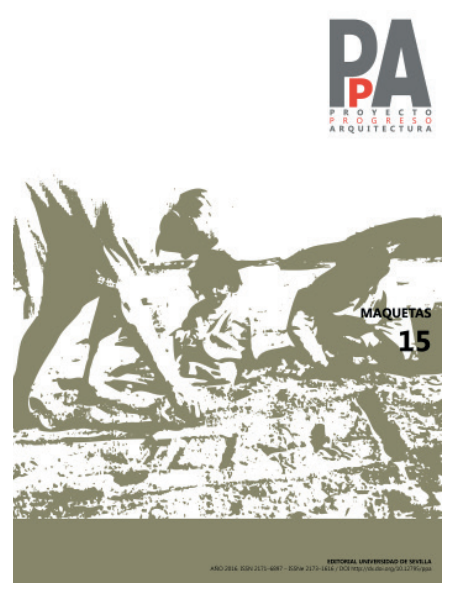

PpA N15
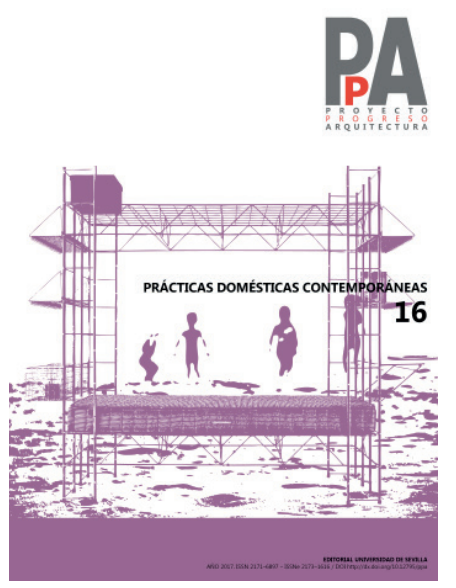

PpA N16

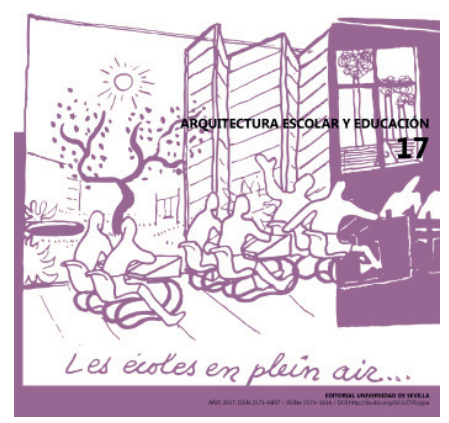

PPA N17

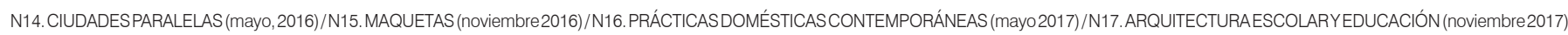

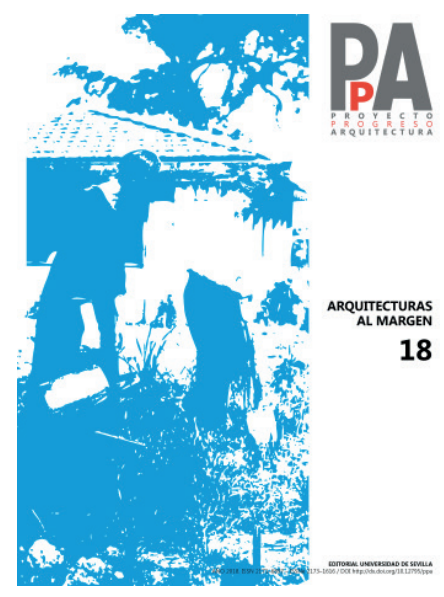

PpA N18

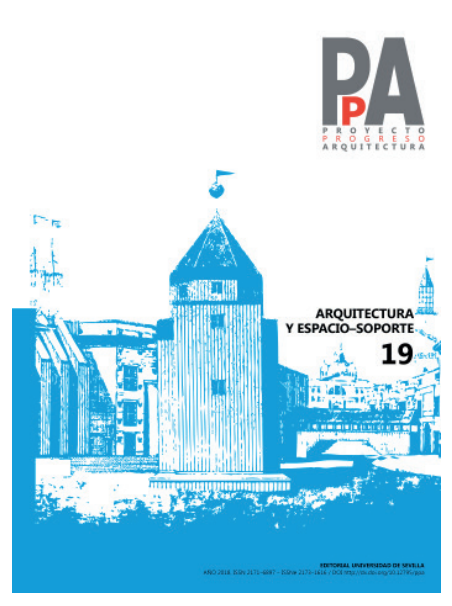

PpA N19

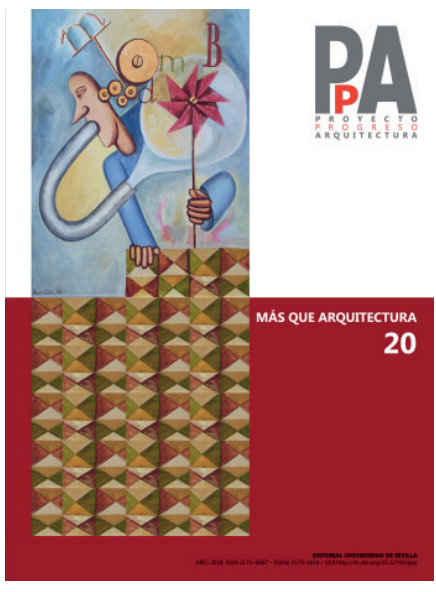

PpA N20

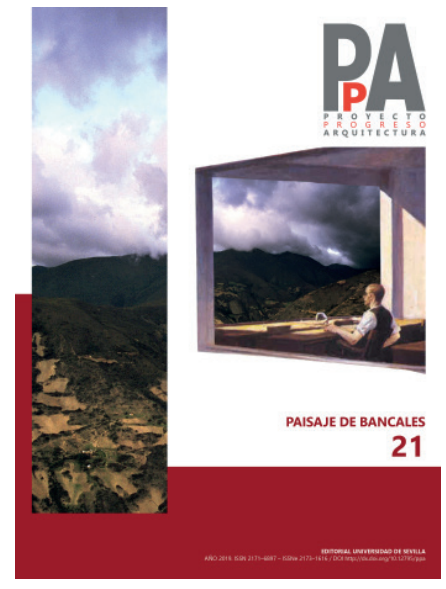

PpA N21

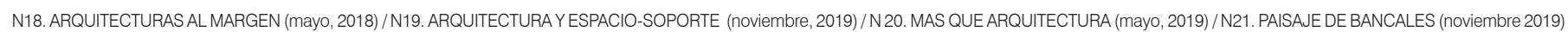

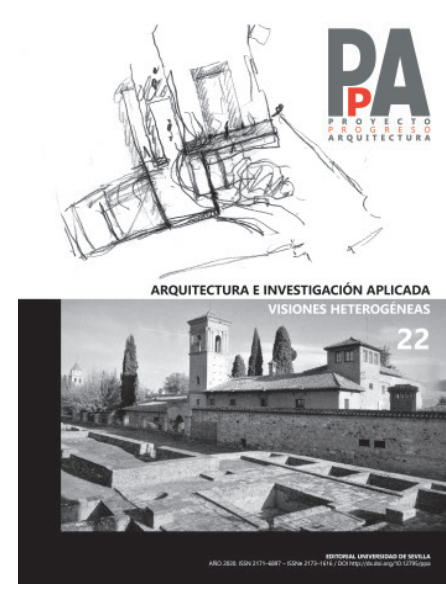

PpA N22

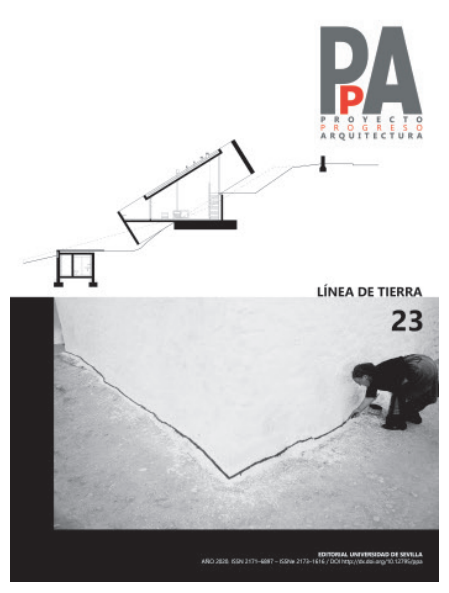

PpA N23
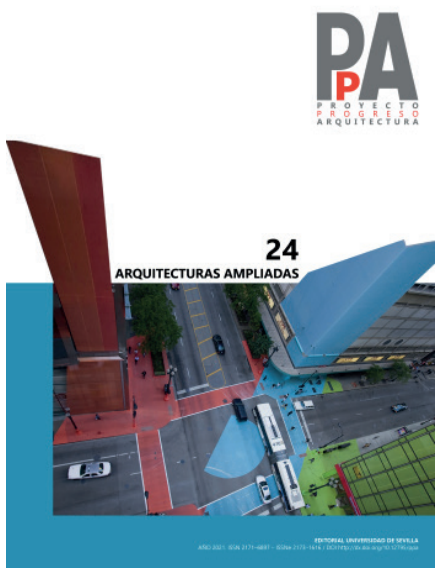

PpA N24

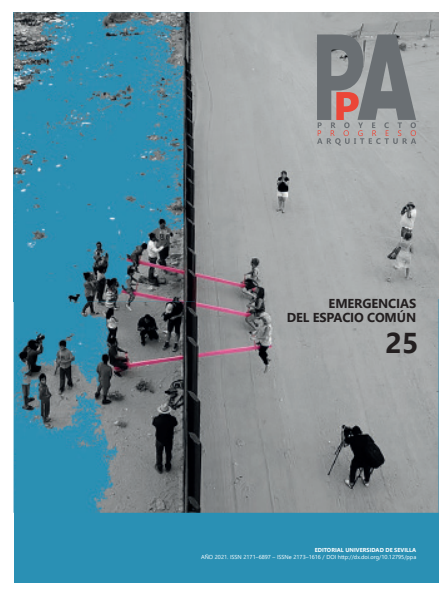

PpA N25

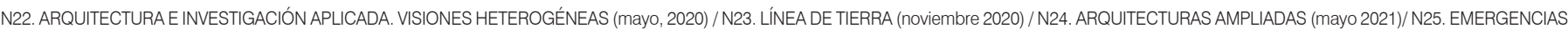
DEL ESPACIO COMÚN (noviembre 2021) 


\section{Autor imagen y fuente bibliográfica de procedencia}

Información facilitada por los autores de los artículos:

página 19, 1. Elaboración propia; página 19, 2. Elaboración propia; página 20, 3. Elaboración propia sobre la base de las fotografías satelitales del Servicio Aerofotográfico Nacional; página 21, 4. Servicio Aerofotográfico Nacional; página 21, 5. Servicio Aerofotográfico Nacional; página 23, 6: Sucesión Emilio Rodríguez Larraín; página $24,7$. Archivo privado Arquitecto Juvenal Baracco; página 25, 8. Archivo privado Arquitecto Juvenal Baracco; página 25, 9. Archivo privado Arquitecto Juvenal Baracco; página 26, 10. Archivo privado Arquitecto Juvenal Baracco; página 27, 11. Elaboración propia; página 28, 12. Fotografía de Jean Pierre Crousse. Archivo Barclay \& Crousse; página 29 , 13. Dibujo de Jean Pierre Crousse. Archivo Barclay \& Crousse; página 30, 14. Fotografía de Eleazar Cuadros. Archivo CCC; página 31, 15. Fotografía de Eleazar Cuadros. Archivo CCC; página 37, 1. The La Guardia and Wagner Archives, La Guardia Community College/The City University of New York; página 38, 2. Cortesía M. Paul Friedberg and Partners. Architectural Record, julio 1966, p. 197; página 39, 3. Cortesía M. Paul Friedberg and Partners. The Architectural Forum, julio-agosto 1966, p. 70; página 41, 4. Elaboración propia; página 42, 5. ๑ David Hirsch. Process: Architecture, 82, p. 31; página 43, 6. @ David Hirsch. Process: Architecture, 82, p. 32; página 43, 7. Cortesía M. Paul Friedberg and Partners. @ David Hirsch; página 44, 8. @ David Hirsch. Process: Architecture, 82, p. 32; página 45, 9. Cortesía M. Paul Friedberg and Partners. ๑ David Hirsch; página 46, 10. ๑ David Hirsch. Process: Architecture, 82, p. 33; página 46, 11. @ David Hirsch. Parks Council Records/Avery Architectural and Fine Arts Library, Columbia University. MOGILEVICH, Mariana. The invention of public space. Minneapolis: University of Minnesota Press, 2020, p. 36; página 46, 12. Cortesía M. Paul Friedberg and Partners. @ David Hirsch; página 47, 13. MOGILEVICH, Mariana. The invention of public space. Minneapolis: University of Minnesota Press, 2020, p. 37; página 48, 14. @ David Hirsch. Process: Architecture, 82, p. 30; página 52, 1. Elaboración propia, composición a partir de fotografías de Îñigo de Barron (izquierda y centro) y Nicolas Vigier (derecha), 2021; página 52, 2. Nicolas Vigier, 2021; página 53, 3. Erich Andres, sin fecha. Ministerio de Educación Cultura y Deporte, Centro Documental de la Memoria Histórica (ES.37274.CDMH//FOTOGRAFíAS-ERICH-ANDRES, 970 y ES.37274.CDMH//FOTOGRAFÍAS-ERICH-ANDRES, 972); página 54, 4. HURTWO0D, Marjory. Why Not Use Our Bomb Sites Like This? En: Picture Post, noviembre 1946, vol. 33, n. ${ }^{16}$, pp. 26-27; página 55, 5. Luis Bartolomé Marcos (LBM1948), 2021. Fotografía publicada en Wikimedia Commons bajo licencia Creative Commons Attribution-ShareAlike 4.0 International: https://creativecommons.org/licenses/by-sa/4.0/deed.en; página 57, 6. Elaboración propia, collage a partir de imágenes intervenidas procedentes de los Amsterdam City Archives, 2020; página 58, 7. Elaboración propia, collage a partir de imágenes procedentes de medios internacionales y redes sociales, 2021; página 59 y 60, Figuras 8 y 9 . Elaboración propia, 2015; página 62, 10: Records of Lady Allen of Hurtwood, Modern Records Centre, University of Warwick (MSS.121-AP-12-17-13). @ Feature Photography: Rosemary Mathews and Howell Evans; página 63, 11. John Drysdale, sin fecha. Records of Lady Allen of Hurtwood, Modern Records Centre, University of Warwick (MSS.121-AP-12-15-3). ๑ Camera Press (Text and Illustrations), London; página 63, 12. John Drysdale, sin fecha. Records of Lady Allen of Hurtwood, Modern Records Centre, University of Warwick (MSS.121-AP-12-15-2). @ Camera Press (Text and Illustrations), London; página 63, 13. Records of Lady Allen of Hurtwood, Modern Records Centre, University of Warwick (MSS.121-AP-12-1-20). @ Associated Press Photo from London; página 65, 14. Cortesía y @ Rael San Fratello, 2019; página 65, 15. RISSELADA, Max; HEUVEL, Dirk van den. Team 10, 1953-1981: In Search of a Utopia of the Present. Rotterdam: NAI Publishers, 2005, pp. 56-57; página 70, 1. Elaboración propia. Imágenes extraídas de Wikipedia (hoja 5/12 del plano de Nolli) y de Venturi, Robert; Scott-Brown, Denise; Izenour, Steven. Learning from Las Vegas. Cambridge: MIT Press, 1977; página 72, 2. Archivo Municipal del Ayuntamiento de Málagá. página 73, 3. Elaboración propia; página 74, 4. Archivo Municipal del Ayuntamiento de Málaga; página 74, 5. Archivo Municipal del Ayuntamiento de Málaga; página 75, 6. Archivo Histórico Provincial de Málaga. Delegación Provincial de Cultura. Signatura 14140/35. Fotografía Leiva; página 77, 7. Elaboración propia; página 78, 8. Elaboración propia; página 79, 9. MORALES FOLGUERA, José Miguel. La Alcazaba de Málaga en el s. xix. Entre la utopía y la realidad. En: Cuadernos de Arte de la Universidad de Granada [en línea]. Granada: Universidad de Granada, noviembre 1984, p. 441. Disponible en: https://revistaseug.ugr.es/index.php/caug/article/view/11109; página 80, 10. Foto Arenas; página 80, 11. Foto Arenas; página 80, 12. Archivo Histórico Fotográfico del Instituto de Estudios Fotográficos de Cataluña (IEFC). Fondo Thomas; página 81, 13. Foto Arenas; página 82, 14. Elaboración propia; página 83, 15. Elaboración propia; página 87, 1. Disponible en https://commons.wikimedia.org/wiki/ File:Untitled_(Xe_Biennale_de_Lyon)_(4103279111).jpg This file is licensed under the Creative Commons Attribution 2.0 Generic license; página 91, 2. Disponible en: https://www.leparisien.fr/seine-saint-denis-93/montreuil-sur-les-traces-de-georges-melies-08-06-2018-7761399.php; página 92, 3. Disponible en: https://www.cairn. info/revue-espaces-et-societes-2011-1-page-15.htm; página 93, 4 y 5. Planos extraídos del texto Les “boutiques" des artisans villageois dans la France d'Ancien Régime (XVle-début du XIXe siècle), escrito por Alain Belmont. En: Annales de Bretagne et des pays de l'Ouest. T. 106, n. ${ }^{\circ}$ 1, 1999.; página 94, 6 y 7. Grabados y dibujos extraídos de VIOLLET-LE-DUC, Eugène. Dictionnaire raisonné de l'architecture française du Xle au XVle siècle/Maison. París: Bance-Morel, ed., 1854-1868. T. 6. La Maison; página 96, 8y 9. ๑ Lacaton \& Vassal, Disponible en: https://www.lacatonvassal.com/index.php?idp=25; página 97, 10 y 11. Disponible en: https://www.inexhibit.com/case-studies/ infinite-places-the-french-pavilion-venice-architecture-biennale-2018/. @ Inexhibit; http://encoreheureux.org/category/news/?lang=en.; página 98, 12. @ La Machinerie. Extraída de VELEZ, Véronique; DEMERY, Cécile; MERCURIO, Céline Di. Etude prospective: Nouveaux usages et modes d'habiter [en línea]. París: L'Union Sociale pour I'Habitat, 2020 [consulta: 07-10-2021]. Disponible en: https://www.union-habitat.org/centre-de-ressources/patrimoine-maitrise-d-ouvrage/nouveaux-usages-et-modesd-habiter-analyse-d; página 99, 13. La Bricothèque; página 99, 14. @ Mon atelier en Ville. Extraídas de VELEZ, Véronique; DEMERY, Cécile; MERCURIO, Céline Di. Etude prospective: Nouveaux usages et modes d'habiter [en línea]. París: L'Union Sociale pour l'Habitat, 2020 [consulta: 07-10-2021]. Disponible en: https://www.union-habitat. org/centre-de-ressources/patrimoine-maitrise-d-ouvrage/nouveaux-usages-et-modes-d-habiter-analyse-d. 

PLAYGROUNDS AND COMMON SPACE. ON PLAYING IN THE SUSPENDED CITY. José Parra-Martínez; Nicolás Stutzin-Donoso; Juan Manuel López-Carreño • LA CALLE COMO ESCENOGRAFÍA URBANA. LAS TRANSFORMACIONES DE LARIOS Y ALCAZABILLA EN EL SIGLO XX / THE STREET AS URBAN SCENOGRAPHY. THE TRANSFORMATIONS OF CALLE LARIOS AND CALLE ALCAZABILLA IN THE TWENTIETH CENTURY. Luisa Alarcón González; Francisco Javier Montero-Fernández • EL BRICOLAJE AL TALLER O EL ESPACIO DE LA TRANSFORMACIÓN EN FRANCIA / FROM DO-IT-YOURSELF TO THE WORKSHOP, OR THE TRANSFORMATION SPACE IN FRANCE. Ophelia Mantz •

- IÑAKI ÁBALOS: PALACIOS COMUNALES ATEMPORALES. GENEALOGÍA Y ANATOMÍA . Daniel Díez Martínez • LE CORBUSIER: ¿CAÑONES, MUNICIONES? ¡GRACIAS! VIVIENDAS... POR FAVOR / JORGE TORRES CUECO Y JUAN CALATRAVA: UNA EXPOSICIÓN, UN PABELLÓN Y UN LIBRO: LE CORBUSIER, $1937-1938$ Ricardo Sánchez Lampreave • VICTORIANO SAINZ GUTIÉRREZ: ALDO ROSSI Y SEVILLA. EL SIGNIFICADO DE UNOS VIAJES. José Ignacio Linazasoro Rodríguez.
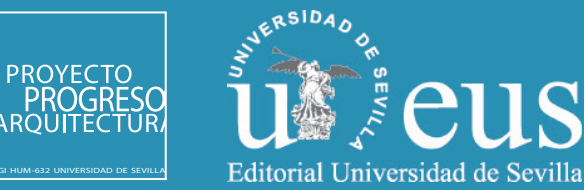